\title{
Profilin 1 deficiency drives mitotic defects and impairs genome stability
}

Federica Scotto di Carlo ${ }^{1}$, Sharon Russo ${ }^{1,2}$, Francesc Muyas ${ }^{3}$, Maria Mangini ${ }^{4}$, Laura Pazzaglia ${ }^{5}$, Flavia Biamonte ${ }^{6}$, Anna Chiara De Luca ${ }^{4}$, Katia Scotlandi ${ }^{5}$, Isidro Cortés-Ciriano ${ }^{3, *}$, Fernando Gianfrancesco $^{1, *}$

${ }^{1}$ Institute of Genetics and Biophysics “Adriano Buzzati-Traverso”, National Research Council of Italy, Naples, Italy; ${ }^{2}$ Department of Environmental, Biological and Pharmaceutical Sciences and Technologies (DiSTABiF), University of Campania Luigi Vanvitelli, Caserta, Italy;

${ }^{3}$ European Molecular Biology Laboratory, European Bioinformatics Institute, Wellcome Genome Campus, Hinxton, $\mathrm{UK}$;

${ }^{4}$ Institute for Experimental Endocrinology and Oncology, "G. Salvatore” (IEOS), Second Unit, Consiglio Nazionale Delle Ricerche (CNR), Napoli, Italy;

${ }^{5}$ IRCCS Istituto Ortopedico Rizzoli, Laboratory of Experimental Oncology, Bologna, Italy;

${ }^{6}$ Department of Experimental and Clinical Medicine, Magna Graecia University, Catanzaro, Italy.

*Address correspondence to: icortes@ebi.ac.uk; fernando.gianfrancesco@igb.cnr.it

\begin{abstract}
Profilin 1 -encoded by PFN1- is a small actin-binding protein with a tumour suppressive role in various adenocarcinomas and pagetic osteosarcomas. However, its contribution to tumour development is not fully understood. Using fix and live cell imaging, we report that Profilin 1 inactivation results in multiple mitotic defects, manifested prominently by anaphase bridges, multipolar spindles, misaligned and lagging chromosomes, and cytokinesis failures. Accordingly, next-generation sequencing technologies highlighted that Profilin 1 knock-out cells display extensive copy-number alterations, which are associated with complex genome rearrangements and chromothripsis events in primary pagetic osteosarcomas with Profilin 1 inactivation.

Mechanistically, we show that Profilin 1 is recruited to the spindle midzone at anaphase, and its deficiency reduces the supply of actin filaments to the cleavage furrow during cytokinesis. The mitotic defects are also observed in mouse embryonic fibroblasts and mesenchymal cells deriving from a newly generated knock-in mouse model harbouring a $P f n l$ loss-of-function mutation. Furthermore, nuclear atypia is also detected in histological sections of mutant femurs. Thus, our results indicate that Profilin 1 has a role in regulating cell division, and its inactivation triggers mitotic defects, one of the major mechanisms through which tumour cells acquire chromosomal instability.
\end{abstract}




\section{Main Text}

Correct chromosome segregation is a fundamental requirement for eukaryotic organisms to maintain genome stability through mitotic divisions ${ }^{1,2}$. The cytoskeleton plays a major role in ensuring faithful chromosome segregation, providing both structural support to maintain cell shape and the mitotic spindle for the movement of the dividing chromosomes ${ }^{3}$. Errors during cell division might generate changes in chromosome content, leading to aneuploidies and polyploidies ${ }^{4}$. Although most aneuploid karyotypes arising during early embryonic life are lethal, aneuploidy occurring in adulthood has been linked to aging and tumorigenesis ${ }^{1,5}$. The ongoing acquisition of alterations in chromosome number and structure is defined as chromosomal instability (CIN), a hallmark of human cancers that contributes to increasing intra-tumour heterogeneity and genomic instability $^{6-8}$. Structural nuclear abnormalities, such as chromosome bridges ${ }^{9-11}$ and micronuclei ${ }^{12}$, are considered morphological markers of $\mathrm{CIN}^{7,13}$ and are associated with defective mitosis. However, our understanding of the molecular mechanisms underpinning aberrant mitosis remains incomplete.

While chromosome alterations are detected in almost all malignant tumours, their prevalence varies across cancers ${ }^{14,15}$. Osteosarcoma (OS) -the most common cancer of bone ${ }^{16}$ represents one of the tumours with the highest degree of genomic instability ${ }^{17,18}$. It mainly affects children and adolescents, where it constitutes a significant cause of cancer-related death ${ }^{16,19-21}$. In elderly patients ( $>60$ years), OS is often secondary to Paget's disease of bone (OS/PDB), and usually presents with a poor prognosis ${ }^{22,23}$. Cancer genome studies revealed that OS are predominantly characterized by a high burden of structural variants (SVs) and complex genome rearrangements ${ }^{17,18,24,25}$. Therefore, recurrent alterations in OS are not frequently detected and clear cancer drivers are not identified. Some molecular understanding about OS development can be gained through well-defined genetic syndromes that show increased risk of OS: germline pathogenic variants have been identified in TP53 (Li-Fraumeni syndrome), RB1 (retinoblastoma), RECQL4 (Rothmund-Thomson syndrome), WRN (Werner syndrome), and BLM (Bloom syndrome $)^{19}$. We recently identified a loss-of-function mutation in the PFN1 gene (c.318_321del) in a hereditary form of OS arising in Paget's disease of bone, and somatic PFN1 loss of heterozygosity $(\mathrm{LOH})$ was also detected in sporadic OS/PDB patients ${ }^{26}$, thus suggesting a potential role for Profilin 1 in OS pathogenesis. PFN1 encodes Profilin 1, the most studied member of a conserved family of four small actin-binding proteins involved in the organisation of the cellular membrane and cytoskeleton $^{27,28}$. Profilin 1 mediates the dynamic remodelling of the actin cytoskeleton by recharging actin monomers with ATP for new filament assembly ${ }^{29}$. Pfn 1-null mice are 
embryonically lethal at the two-cell stage, indicating that Profilin 1 is essential for cell division and survival $^{30}$. In contrast, Profilin 1 is downregulated in various adenocarcinomas (breast ${ }^{31-33}$, hepatic $^{34}$, pancreatic ${ }^{35,36}$, and bladder ${ }^{37}$ ), which correlates with increased metastatic potential and shorter overall survival ${ }^{36}$, suggesting a dosage-dependent effect on a fine-tuned balance between cellular lethality and neoplastic transformation. Although low levels of Profilin 1 have been associated with disruption of cytokinesis and impairment of the contractile ring in both Drosophila melanogaster $^{38}$ and mouse ${ }^{39}$ models, whether this could represent the underlying mechanism for tumour onset has not been investigated. Accurate coordination of the assembly/disassembly of the actin cytoskeleton is a crucial requirement to shape cells, especially to guide mitosis $\mathrm{s}^{3,40}$. We therefore hypothesised that the reduction of Profilin 1 observed in OS and adenocarcinomas underpins tumour onset due to mitotic errors.

In this study, we show that reduced Profilin 1 levels drive mitotic defects that foster CIN, as indicated by the frequent formation of micronuclei, chromosome bridges, and lagging and misaligned chromosomes. Profilin 1 deficiency results in extensive somatic copy-number alterations (SCNAs) in a mesenchymal cellular model. In addition, genome sequencing of OS/PDB tumour biopsies with PFN1 loss of heterozygosity shows complex genome rearrangements, including chromothripsis. Together, our results show that reduced Profilin 1 levels correlate with increased cell division defects that promote genomic instability. 


\section{Results}

Profilin 1 localises to the spindle midzone to promote actin accumulation at the cleavage furrow

To determine the role of Profilin 1 in cell division, we analysed its subcellular localisation during different stages of mitosis. For this purpose, we used human RPE1 cells, which are diploid, non-transformed, hTERT-immortalised retinal pigment epithelial cells widely used as a model to study mitosis ${ }^{41-45}$. Wild type (WT) RPE1 cells were synchronised, immunostained and analysed throughout mitosis, from prophase to near completion of cytokinesis (Methods). Profilin 1 initially showed a uniform distribution throughout the cytoplasm, and then accumulated between the condensed chromosomes in metaphase (Fig. 1). In anaphase, an intense signal at the central region of the spindle became visible, indicating strong recruitment of Profilin 1 from the cytoplasmic pool to the spindle midzone, which decreased in late anaphase and telophase (Fig. 1). The mitotic localisation of Profilin 1 was also analysed in three heterozygous and three homozygous PFN1 knock-out (KO) RPE1 clones, which we generated using CRISPR/Cas9 (Supplementary Fig. 1a,b; Methods). $P F N 1^{+/-}$RPE1 clones manifested a general reduction of Profilin 1 expression, and lacked an overt enrichment of Profilin 1 in the midzone; whereas $P F N 1^{-/-}$cells were expectedly devoid of Profilin 1 in the cytoplasm (Fig. 2a). The spindle midzone is required to properly position the cleavage furrow, whose ingression is mediated by contractile forces generated by filaments of actin and $\operatorname{myosin}^{46,47}$. Therefore, we investigated the impairment of the contractile ring in PFN1$\mathrm{KO}$ cells by examining actin nucleation at the cleavage furrow of $P F N 1^{+/}$and $P F N 1^{-/}$RPE1 cells through phalloidin staining. Of note, the fluorescence intensity of F-actin within the cleavage furrow of $P F N 1^{+/-}$and $P F N 1^{-/-}$cells was markedly decreased compared with WT RPE1 $(P=0.0004$ and $P<0.0001$, respectively; one-way ANOVA), demonstrating the relevance of Profilin 1 in regulating actin polymerisation at specific stages of mitosis (Fig. 2b,c).

\section{Lack of Profilin 1 triggers prolonged mitosis due to impaired mitotic entry and exit}

To determine the effect of Profilin 1 deficiency on mitosis, we used time-lapse phasecontrast light microscopy (Methods). We followed fields of WT and KO RPE1 cells for 15-20 h after thymidine synchronisation at early S phase and subsequent release (Fig. 3a). We observed that, while $65.3 \pm 4.8 \%$ of WT cells committed to mitosis after $\sim 5 / 6 \mathrm{~h}$ following release, only 13.0 $\pm 6.5 \%$ of $P F N 1^{+/}$and $24.7 \pm 3.8 \%$ of $P F N 1^{-/}$RPE1 entered mitosis within $\sim 10 / 12 \mathrm{~h}$ from release (Fig. 3b). In addition, while normal cells took 18.5 minutes (95\% Confidence Interval (CI): 17.5 - 
19.4) from mitotic rounding to cytokinesis, $\mathrm{KO}$ cells spent 30.1 minutes (95\% CI: 26.1 - 34.0) in mitosis before completing cell division (Fig. 3c,d; Supplementary Videos 1-3). We also observed cases in which mutant cells underwent cytokinesis failure ( 5 of 66 mitoses of $P F N 1^{+/-}$and 3 of 42 mitoses of $\mathrm{PFN1}^{-/-} \mathrm{RPE} 1$ ), resulting in a doubled genome in the subsequent interphase, which was never detected in 127 mitoses of WT cells (Supplementary Fig. 2a). These results are in agreement with the lower amount of actin at the contractile ring in PFN1 mutant cells (Fig 2b,c). Upon transition to mitosis, cells reduce their adhesions to the substrate and round up through the formation of a dense cortical actin network ${ }^{3}$. Interestingly, we observed that PFN1-KO cells were able to disassemble focal adhesions only partially, acquired an incomplete spherical shape and failed to completely round up. Some cells ended up succeeding cell division, yet most cells took several attempts to initiate the M phase without effectively dividing during the 15-20 h of timelapse observation (Supplementary Fig. 2b; Supplementary Videos 1-3). The difficulty of KO cells to acquire a spherical shape let us speculate that the lack of cell rounding could be the consequence of insufficient levels of cortical $\operatorname{actin}^{48,49}$. Fluorescent staining of F-actin revealed that the intense circular band of actin in control mitotic cells was decreased in $P F N 1^{+-}(P=0.0002$, one-way ANOVA) and $P F N 1^{-/}$cells $(P<0.0001$, one-way ANOVA) (Fig. 3e,f). Of note, we observed a remarkable difference in cell shape in metaphase, with KO RPE1 unable to achieve a complete rounding and exhibiting an irregular shape, whereas rounded metaphase cells were found in control cultures (Fig. 3e). These data indicate that Profilin 1 loss dramatically harms the morphology of mitotic cells, compromising mitotic rounding and cytokinesis, and delaying the overall cell division process.

\section{Profilin 1-dependent mitotic delay is accompanied by chromosome segregation defects}

To investigate the effect of Profilin 1 loss on chromosome segregation, we determined the fraction of mitotic WT and PFN1-KO RPE1 cells with chromosome mis-segregation events after thymidine synchronisation and release through confocal microscopy analysis of cells with immunostained microtubules (Fig. 4a; Methods). Both $P F N 1^{+-}$and $P F N 1^{-/-}$RPE1 cells displayed chromosome segregation defects at higher frequency than the parental cell line (Fig. 4b-i;

Supplementary Table 1). Remarkably, a higher frequency of chromosome misalignments at the metaphase plate was observed in $P F N 1^{+/-}\left(P=0.0096\right.$, one-way ANOVA) and $P F N 1^{-/-}$RPE1 cells $(P=0.0411$, one-way ANOVA) compared to WT (Fig. 4b,f). Normal spindle assembly was also disrupted with increased formation of multipolarity in $P F N 1^{+/-}(P=0.0305$, one-way ANOVA $)$ and $P F N 1^{-/-}$cells $(P=0.0259$, one-way ANOVA) (Fig. 4c,g). Furthermore, evaluation of microscopy 
images of anaphases and telophases revealed that $\mathrm{PFN1}^{+/-}$and $P F N 1^{-/-}$cells were more prone to chromosome bridges ( $P=0.0003$ and $P=0.0009$, respectively; one-way ANOVA) (Fig. 4d,h; Supplementary Fig. 3) and contained lagging chromosomes at a higher frequency than control cells $(P=0.2457$ and $P=0.0345$, respectively; one-way ANOVA) (Fig. 4e,i). The frequency of nuclear atypia detected in homozygous $\mathrm{KO}$ clones and heterozygous clones was similar $(P>0.05$, Student's $t$-test), indicating that the loss of one PFN1 functional copy is sufficient to induce CIN (Supplementary Table 1). Together, these results show that Profilin 1 insufficiency destabilises the regulation of mitosis, leading to chromosome segregation abnormalities during cell division.

To rule out the possibility that the formation of abnormal nuclear structures could be an unintended off-target effect of CRISPR/Cas $9^{50,51}$, we analysed mitotic RPE1 cells stably expressing either non-target or PFN1-shRNA (Supplementary Fig. 4a; Methods). Anaphase bridges were the most common defects, and the knockdown of PFN1 triggered their formation at a higher frequency as compared to cells expressing the control shRNA $(P=0.0041$, Student's $t$-test) (Supplementary Fig. 4b). Additionally, $3.0 \pm 0.9 \%$ of anaphase/telophase cells contained lagging chromosome fragments and, rarely ( 2 out of 307), metaphase plates showed misaligned chromosomes (Supplementary Fig. 4c), which were not found in control cells $(n=183)$.

To follow the fate of daughter cells with mis-segregated chromosomes, we performed fluorescence live cell imaging of WT, $P F N 1^{+/}$and $P F N 1^{-/-}$RPE1 cells stably expressing H2BmCherry/EGFP-Tubulin (Methods). The elevated occurrence of mitotic aberrations was confirmed in $\mathrm{PFN1}^{+/-}$and $\mathrm{PFN1}^{-/-}$RPE1 cells, namely chromosome misalignment on metaphase plates, anaphase bridges, micronuclei formation, and cytokinesis failures (Supplementary Fig. 5). Although we noted floating apoptotic cells in KO cultures, these mitotic errors resulted in viable daughter cells in most cases, suggesting that a p53-deficient background would enhance mitotic dysfunction and tumorigenicity ${ }^{52-55}$ (Supplementary Videos 4-7).

\section{Profilin 1 insufficiency prompts mitotic errors in mesenchymal cells and in vivo}

Loss of function of PFN1 was previously detected in $\mathrm{OS} / \mathrm{PDB}^{26}$, an aggressive tumour of mesenchymal origin. Therefore, to investigate the role of Profilin 1 in the maintenance of genome integrity also in mesenchymal cells, we analysed $P f n 1-\mathrm{KO}$ MC3T3 mouse cells that we engineered to obtain heterozygous $\mathrm{KO}$ clones $^{26}$. We confirmed the impairment of cell division as a consequence of Profilin 1 loss, since $50.7 \pm 5.0 \%$ of WT MC3T3 cells completed mitosis in the time-frame of observation versus $30.5 \pm 4.9 \%$ of $P f n 1^{+/-}$MC3T3 cells (Supplementary Fig. 6a,b). 
Mutant cells took on average 30.8 minutes (95\% CI: 28.4 - 33.1) to divide, whereas control cells spent an average of 24.5 minutes (95\% CI: 22.8 - 26.1) in mitosis (Supplementary Fig. 6c,d; Supplementary Videos 8,9). We then evaluated the rate of nuclear abnormalities in $P f n 1^{+/}$MC3T3 as compared to WT MC3T3 cells. Fluorescent staining of interphase nuclei revealed an increased frequency of nuclear abnormalities in mutant cells, namely binucleation $(P=0.0004$, Student's $t$ test) (Fig. 5a,d), chromosome bridges ( $P=0.0021$, Student's $t$-test) (Fig. 5b,e), and spontaneous formation of micronuclei ( $P=0.0049$, Student's $t$-test) (Fig. 5c,f). To assess whether micronucleation was related to DNA damage, we measured the damage-dependent phosphorylation of the histone variant $\mathrm{H} 2 \mathrm{AX}(\gamma-\mathrm{H} 2 \mathrm{AX})$. We identified $\gamma-\mathrm{H} 2 \mathrm{AX}$ positive-micronuclei in $67.2 \pm 5.6 \%$ of $P f n 1^{+/}$cells, indicating that mutant micronuclei harboured DNA double strand breaks (DSBs) (Fig. 5g,h). By contrast, only a few micronucleated WT cells $(6.3 \pm 2.4 \%)$ were positive for $\gamma$ H2AX (Fig. 5h). In the absence of cell stress, p53 is maintained at low levels; however, in the presence of DNA damage, it rapidly accumulates within the cell nucleus ${ }^{56,57}$. Therefore, to test whether the DSBs observed in $P f n 1^{+/-}$cells activated a p53-mediated response, we measured p53 levels in WT and Pfn ${ }^{+/-}$MC3T3 cells. We found that p53 levels increased concomitantly with the reduction of Profilin 1 (Fig. 5i,j). As expected, p53 accumulation was only detected in the nuclei of $P f n 1^{+/-}$MC3T3 cells, especially in the cells showing micronuclei and chromosome bridges (Fig. $5 \mathbf{k})$.

To assess the consequences of Profilin 1 inactivation in vivo, we generated a mouse model harbouring the $P f n l$ loss-of-function mutation previously identified in OS/PDB (c.318_321del) ${ }^{26}$ (Supplementary Fig. 7; Methods). Homozygous Pfnl knock-in mice died in utero; therefore, we isolated mouse embryonic fibroblasts (MEFs) at 14.5 days post coitum in an attempt to track cell proliferation in WT and mutant cells. However, homozygous ( $\left.P f n 1^{c .318 \_321 d e l / c .318 \_321 d e l}\right)$ embryos were not found even at this stage $(n=44$ embryos), nor did females present resorbed decidua in the uterus, suggesting that $P f n 1$ is essential for the earliest cell divisions in mouse embryos. Time-lapse phase-contrast microscopy of control $\left(P f n 1^{W T / W T}\right)$ and heterozygous knock-in $\left(P f n 1^{c .318 \_32 l d e l / W T}\right)$ MEFs confirmed a high rate of mitotic defects in mutant cells, especially failures in mitotic rounding and cytokinesis (Supplementary Videos 10,11). We next validated this finding in mesenchymal cells isolated from calvarias of viable heterozygous newborns. Confocal analysis of nuclear shape revealed that $P f n 1^{c .318 \_321 d e l / W T}$ mesenchymal cells showed an increased frequency of micronuclei formation as compared to $P f n 1^{W T / W T}$ cells $(P=0.0312$, Student's $t$-test $)$ (Fig. 6a,b). Although the frequency of binucleation was not significantly higher in $P f n 1^{c .318 \_321 d e l / W T}$ cells compared with WT $(P=0.0778$, Student's $t$-test) (Fig. 6c), we occasionally observed knock-in cells with three nuclei, which were never observed in control cell cultures (Fig. 6d). These results 
suggest that replication defects were more penetrant in the presence of the heterozygous loss-offunction mutation.

Given the frequent occurrence of OS in the epiphysis of long bones ${ }^{16}$, we examined the growth plate of femurs of 4-month-old mice for the presence of aberrant mitoses. Interestingly, the analysis of the histological sections highlighted a 5-fold increase in the frequency of abnormally binucleated cells $(P=0.0054$, Student's $t$-test $)$ and rare trinucleated cells $(P=0.0532$, Student's $t$ test) in Profilin 1-deficient growth plates compared with cells in femurs from WT mice (Fig. 6e-g), which is in line with the observed defective cytokinesis in PFN1-KO RPE1 cells (Supplementary Figure 2 and Supplementary Figure 5) and in $P f n 1^{c .318 \_321 d e l / W T}$ MEFs (Supplementary Video 11). Collectively, these results indicate that reduced levels of Profilin 1 are sufficient to induce mitotic errors that result in nuclear abnormalities also in vivo.

\section{High frequency of genomic rearrangements in cellular models and pagetic osteosarcoma}

Osteosarcoma is a mesenchymal tumour characterised by extensive somatic copy-number aberrations (SCNAs) and SVs ${ }^{17,18,24}$. To test whether the nuclear abnormalities related to PfnI haploinsufficiency effectively resulted in chromosome copy-number changes, we performed lowpass whole-genome sequencing (WGS) on 8 different single-cell-derived $P f n 1^{+/-}$MC3T3 clones and the WT culture (Methods). We first confirmed that all MC3T3 clones were efficiently knocked out through Western blot, Sanger sequencing analysis, and WGS (Supplementary Fig. 8a,b). Copynumber analysis revealed that all $P f n 1^{+/-}$MC3T3 clones harboured at least one chromosomal alteration compared to WT MC3T3 cells (range: 2-13; Supplementary Fig. 9). This result suggests that the loss of $P f n 1$ results in replication defects and consequent accumulation of SCNAs.

We next analysed human tumours to assess whether complex patterns of genome instability, such as chromothripsis, are present in OS/PDB genomes. To this aim, we performed WGS on paired tumour and matched normal samples for 4 primary OS tumours from PDB patients as well as whole-exome sequencing (WES) for 10 additional tumours (mean age at diagnosis 66 years old; 95\% CI: 62 - 71) (Fig. 7; Supplementary Table 2; Methods). The 4 WGS samples had polyploid genomes (ploidy $\geq 2.5$ ) and a high number of SCNAs and SVs (range 202-324 SVs) (Fig. 7a;

Supplementary Table 2), including chromothripsis affecting a single (chromosome 8 in patient 1798; Fig. 7a) or multiple chromosomes (e.g., chromosomes 5 and 13 in patient 1363) accompanied by high-level copy-number amplifications. Extensive loss of heterozygosity (LOH) across multiple 
chromosomes was frequently observed (Fig. 7b,c), and we detected LOH of PFN1 in 3 out of 4 WGS samples, and in 7 out of 10 WES samples (Supplementary Table 2).

Copy-number analysis revealed that 8/14 tumours had undergone whole-genome doubling (WGD) events, with 3 samples undergoing WGD twice (Fig. 7d; Methods). Next, we sought to investigate when WGD events occurred during tumour evolution. To this aim, we integrated somatic point mutation and copy-number data to perform both relative and real-time timing analysis of WGD events (Methods) ${ }^{58,59}$. By assuming a constant rate of mutation accumulation during tumour evolution, which allows to perform real-time estimates for WGD events relative to the age at diagnosis ${ }^{59}$, we found that WGD events occurred decades before diagnosis in the 4 patients (ranging from 20 to 48 years before diagnosis), and in the 2 cases with 2 WGD events, the estimated time between doublings was also in the order of decades (Fig. 7e; Supplementary Table 2).

Together, these data indicate that OS in PDB patients shows remarkably complex genome aberrations, including WGD events and chromosomal rearrangements. 


\section{Discussion}

Animal cells undergo dramatic morphological changes as they progress through the different stages of mitosis ${ }^{3}$. The actin cytoskeleton contributes to spindle morphogenesis and positioning ${ }^{60-62}$ and thus, deregulation of actin dynamics could jeopardise chromosome segregation. The mitotic spherical shape is achieved through an increase in the cortical tension at prophase, triggered by RhoA-mediated phosphorylation of DIAPH1, which utilises Profilin 1 to polymerise actin at the cortex $^{63}$. Therefore, we here hypothesised that Profilin 1 inactivation, which has been observed in diverse types of carcinomas ${ }^{31-37}$ and sarcomas ${ }^{26,64}$, might result in cytoskeletal defects that lead to CIN. We used both CRISPR/Cas9 and shRNA experiments to inactivate PFN1 expression in RPE1 cells, which are widely used to study mitotic defects as well as chromosomal rearrangements in a p53-deficient background ${ }^{12,65-67}$. Our data show that Profilin 1 contributes to mitotic cell rounding, since Profilin 1-deficient cells struggle to round up, and the transition from G2 to mitosis requires several attempts, delaying cell division. The observed defects in mitotic cell rounding were accompanied by a significant reduction in cortical actin and, more importantly, chromosome missegregation events. The loss of chromosome segregation fidelity induces $\mathrm{CIN}^{2}$, a hallmark of many tumours and especially osteosarcomas ${ }^{7,17,18,25}$. Consistent with this, mitotic dysfunction and SCNAs were also detected in mesenchymal cells depleted for $P f n 1$, both in vitro (MC3T3) and ex vivo (MEFs and calvaria-derived cells).

Heterozygous $P f n l$ knock-in mice display no overt tumour phenotype. Nonetheless, the evidence that haploinsufficiency of $P f n l$ did not drive tumorigenesis within 18 months of age is not surprising, mainly in animals with functional p53. Indeed, haploinsufficiency for most of the genes involved in the maintenance of chromosome stability (e.g. Cenpe, Mad1, Mad2, Plk4, Bubl) leads to long tumour latency and low penetrance, despite the high percentage of aneuploid cells ${ }^{68-73}$, suggesting that there are factors (e.g. p53) that restrain the transforming potential of aneuploidy ${ }^{73,74}$. Here, we found that Profilin 1 deficiency triggered the activation of the p53 pathway, and floating dead cells were observed in PFN1-KO cell cultures, likely as a result of p53-induced apoptosis. Nevertheless, our results demonstrate that most abnormal mitoses did result in viable daughter cells, implying that Profilin 1 inactivation causes mitotic errors even in a p53-proficient background.

In all cell models analysed, we pointed out occasional cytokinesis defects resulting in binucleated daughter cells. This result could be explained by insufficient accumulation of F-actin in the cleavage furrow of PFN1-KO RPE1 cells as a result of poor recruitment of Profilin 1 to the spindle midzone. This result is consistent with previous findings showing frequent binucleation in chondrocytes from mice conditionally depleted for $P f n 1$ in cartilage ${ }^{39}$. While cytokinesis is essential for cell proliferation, an important fraction of cancers likely result from a cytokinesis failure ${ }^{75,76}$. 
Accordingly, WGD events and LOH at the PFN1 locus were frequently detected in OS/PDB tumours. Our analysis revealed that PFN1 loss occurred before WGD, which could result from cytokinesis failures induced by Profilin 1 reduction. In addition to WGD, we observed extensive SCNAs and massive genomic rearrangements in OS/PDB samples, including chromothripsis. Although we cannot causally link the presence of complex rearrangements and loss of PFN1 in clinical samples given that TP53 is also inactivated in these cases, our results are in line with the high frequency of chromosome bridges and micronuclei found in Profilin 1-deficient cells, both reported to contribute to genome instability and chromothripsis ${ }^{67,77,78}$.

In conclusion, we propose that Profilin 1 inactivation results in defective cytoskeleton organisation, thereby affecting mitotic entry, progression, and exit. Our work mechanistically links Profilin 1-dependent mitotic defects to CIN in epithelial and mesenchymal cell lines, and mouse experiments. By uncovering mitotic defects without inactivating p53, we here also highlight PFN1 knock-out as a tool to study aneuploidy and complex genome rearrangements in a p53-proficient background. 


\section{Methods}

Cell culture and treatment. All cell lines were maintained in a humidified incubator at $37{ }^{\circ} \mathrm{C}$ with 5\% $\mathrm{CO}_{2}$. hTERT-RPE1 (referred to as RPE1) and derivatives were grown in DMEM/F12 (Thermo Fisher, 11320033) supplemented with 10\% FBS and $0.01 \mathrm{mg} / \mathrm{ml}$ Hygromycin B (Thermo Fisher, 10687010); RPE1-derived shRNA clones were cultured in the presence of $0.4 \mathrm{mg} / \mathrm{ml}$ neomycin. To synchronise RPE1 cells, cells were treated with $5 \mathrm{mM}$ thymidine (Sigma-Aldrich, T1895) for $16 \mathrm{~h}$, released for $8 \mathrm{~h}$, and then treated again with thymidine for $16 \mathrm{~h}$. After 2 washes with 1x PBS (Thermo Fisher, 14190169), cells were cultured in standard DMEM/F12 medium until entering mitosis, fixed and subjected to immunofluorescence experiments. Based on our experience, WT and CRISPR-WT RPE1 cells accumulate at M phase after a 7-h release into normal medium, $P F N 1^{+/-}$ RPE1 cells enter M phase after 8/9-h release, whereas the majority of $P F N 1^{-/-}$RPE1 cells peak at mitosis after 12-14 h of release. In live cell imaging experiments, cells were recorded after 2 hours from the second release in thymidine-free medium. MC3T3 and derivatives were maintained in Minimum Essential Medium $\alpha$ (MEM- $\alpha$ ) without ascorbic acid (Thermo Fisher, A1049001) with $10 \%$ FBS, $1 \%$ penicillin-streptomycin, and $0.5 \%$ gentamycin. To synchronise MC3T3 cells in early $\mathrm{S}$ phase, cells were cultured for $30 \mathrm{~h}$ in $2 \mathrm{mM}$ thymidine, washed 2 times in 1x PBS and released into the standard MEM- $\alpha$ medium; after 2 hours, cells were subjected to live cell imaging. HEK293T (293T) were cultured in DMEM High-Glucose (Thermo Fisher, 11965084) with 10\% FBS and 1\% penicillin-streptomycin. All cell lines were tested for mycoplasma contamination (PCR Mycoplasma Detection Kit, Applied Biological Materials, G238) and were found to be negative. Cell lines were not authenticated.

Generation of a Pfn1 knock-in mouse model. The 129/Sv genomic DNA was used as a template to amplify the $P f n 1$ locus for the homologous recombination. The Pfn1 c.318_321delTGCC mutation was introduced in the targeting vector by PCR-mediated mutagenesis (QuikChange Lightning, Agilent). Targeting of the construct was done into the E14Tg2a embryonal stem cell line, and targeted ES cell clones were identified by Southern blot analysis using a 3 ' probe and an internal Neo probe on EcoRI-cut genomic DNA, and a 5' probe on BamHI-cut genomic DNA. One $P f n 1^{\mathrm{c} .318 \_321 \mathrm{del} / \mathrm{WT}}$ embryonic stem cell clone was injected into C57BL6 blastocysts to establish a mutant mouse colony. These mice were maintained on a mixed genetic background. Genotyping was performed with allele-specific primers: forward 5'-AACTCCAGCTCCACAGTACATAAG-3'; reverse 5'-TTATGCAGCCTTGACACTGAGGAC-3'. Heterozygous mutant crosses did not yield any homozygous mutant animals at birth $(n>200$ live births) or as embryos $(n=44)$. Animals were 
handled in accordance with the authorization no. 125-2021-PR released by the Italian Ministry of Health; all mice were housed in a pathogen-free barrier environment.

Isolation of mouse embryonic fibroblasts. $P f n 1^{c .318 \_321 d e l / W T}$ heterozygous mice were crossed; pregnant females were sacrificed at 14.5 days after the appearance of the copulation plug by cervical dislocation. Uterine horns were dissected out and placed in tubes containing PBS $+1 \%$ penicillin-streptomycin. Embryos were separated from their placenta and surrounding membranes. Red organs (heart and liver) and tail (for genotyping) were removed. Embryos were finely minced using razor blades and suspended in a tube containing $3 \mathrm{ml} 0.05 \%$ trypsin and incubated at $37{ }^{\circ} \mathrm{C}$ for $15 \mathrm{~min}$ in a shaking water bath. Two volumes of media (DMEM supplemented with $10 \%$ FBS, $1 \%$ penicillin-streptomycin) were added to inactivate the trypsin, and remaining pieces were removed by letting them to settle down to the bottom of the tube within $5 \mathrm{~min}$. The supernatant was carefully taken off to be subjected to low-speed centrifugation. Cell pellet was resuspended in warm medium and plated in $25 \mathrm{~cm}^{2}$ flasks.

Isolation and culture of neonatal mouse calvaria-derived cells. Primary murine mesenchymal stromal cells (mMSCs) were isolated from neonatal mouse calvarias. Mice pups (P2/3) were euthanized by inhalation of $\mathrm{CO}_{2}$, heads from $P f n 1^{W T / W T}$ and $P f n 1^{c .318 \_321 d e l / W T}$ were individually placed in a petri dish with 1x PBS, calvaria were cleaned from adherent soft tissues and subjected to sequential digestion in 1x PBS containing $1 \mathrm{mg} / \mathrm{ml}$ collagenase type IV (Sigma-Aldrich, C5138), $0.025 \%$ trypsin (Biowest, L0909) and $1 \%$ penicillin-streptomycin, at $37{ }^{\circ} \mathrm{C}$ in shaking water bath. The cells from digestions 2-4 were collected, pooled and cultured in MEM- $\alpha$ without ascorbic acid with $10 \%$ FBS and 1\% penicillin-streptomycin; cells were maintained in culture for no more than 4 passages.

Targeted knock-out of PFN1 using CRISPR-Cas9. PFN1-KO RPE1 cell clones were generated using the CRISPR guide RNA (gRNA) 5'- CACCGTTCGTACCAAGAGCACCGGT-3'. Annealed gRNA oligonucleotides were inserted into the pSpCas9n(BB)-2A-GFP plasmid (Addgene, PX458) and the construct was transfected into RPE1 cells using Lipofectamine LTX with PlusReagent (Invitrogen) transfection reagent. GFP-positive single cells were sorted by FACS into 96-well plates. Candidate single-clone colonies were verified by Sanger sequencing and western blotting. Off-target mutations in genes predicted by Optimized CRISPR design tool at http://crispr.mit.edu/ (GRAMD4, INTS6) were excluded through Sanger sequencing. 
PFN1 silencing. To knockdown Profilin 1 expression in RPE1 cells, a custom pLKO.1-puro-CMVTurboGFP was purchased from Sigma-Aldrich, expressing a shRNA previously shown as efficiently targeting the CDS of PFN1 (5'-

\section{CCGGCGGTGGTTTGATCAACAAGAACTCGAGTTCTTGTTGATCAAACCACCGTTTTT-3’)}

${ }^{79}$. As a negative control, the same pLKO-based plasmid expressing a non-target shRNA was purchased from Sigma-Aldrich and used. Stable RPE1 clones were generated through Nucleofection using the Amaxa Cell Line Nucleofector Kit V (VCA-1003), following the X-001 protocol provided by Amaxa Nucleofector (Lonza). After $24 \mathrm{~h}$, clones stably expressing either the non-target or the PFN1 shRNA were selected with neomycin $(0.5 \mathrm{mg} / \mathrm{ml})$ every $60 \mathrm{~h}$ for 10 days. Single cell clones were picked and expanded; colonies from a single cell were then maintained with $0.4 \mathrm{mg} / \mathrm{ml}$ neomycin. Silencing was verified through western blotting. Three non-target (shControl) and five shPFN1 RPE1 clones were subjected to the experiments.

Lentiviral infection. Stable H2B-mCherry/EGFP-Tubulin RPE1 cells were generated by lentiviral transduction. Lentiviral plasmids expressing H2B-mCherry or EGFP-Tubulin were purchased by Addgene (CSII-prEF1a-mCherry-3xNLS, \#125262; L304-EGFP-Tubulin-WT, \#64060). pCMVVSV-G and pCMV- $\triangle$ R8.2 co-packaging plasmids were a gift from Dr Annalisa Fico. Lentiviruses expressing the respective genes were generated by cotransfection of 293T cells with pCMV-VSV-G and pCMV- $\triangle \mathrm{R} 8.2$, using Lipofectamine 2000 (Invitrogen). The lentiviral supernatant was collected after 24 and 48 hours, and then used for infection of target cells. Infected cells ( $5 \times 10^{4}$ ) exhibiting both mCherry and GFP fluorescence were selected through FACS Aria (Becton Dickinson) and further expanded.

Time-lapse microscopy. For live cell imaging, RPE1, MC3T3, and MEF cells were cultured as described above. For phase-contrast time-lapse analysis, RPE1 and MC3T3 were synchronised at the G1/S boundary by the thymidine block method, as described previously. At $2 \mathrm{~h}$ from the release in thymidine-free medium, the cells regained the cell cycle progression in a microscope stage incubator at $37{ }^{\circ} \mathrm{C}$ in a humidified atmosphere of $5 \% \mathrm{CO}_{2}$ throughout the experiment. MEF cells were analysed as asynchronous population due to the accumulation of senescent cells observed in thymidine-treated MEF cultures. Cells were cultured in 6-well plates $\left(4 \times 10^{4} \mathrm{RPE} 1 ; 6 \times 10^{4}\right.$ MC3T3; 1.2 x 10 $0^{5}$ MEFs). Time-lapse images were acquired with an inverted Zeiss Axio Observer Z1 widefield microscope equipped with an AxioCam MRm grayscale CCD camera and controlled 
by ZEN pro software (Zeiss). Phase-contrast images were captured as Z-stacks (10 planes, $3 \mu \mathrm{m}$ interval) every 2 min for 15-20 h, using a x20 objective. The resulting images were processed and analysed using ZEN pro (Zeiss) software. For fluorescence live cell imaging, 6 x $10^{4}$ asynchronous H2B-mCherry/EGFP-Tubulin RPE1 cells were plated on a 6-well plate 20 hours before the acquisition, and then placed on the stage of a THUNDER Imager Live Cell (Leica) equipped with an environmental chamber which provided an adequate temperature, humidity, and $\mathrm{CO}_{2}$ control. Time-lapse images were captured as Z-stacks (12 planes, $2.4 \mu \mathrm{m}$ interval) at 3 positions every 2 min for $\sim 20 \mathrm{~h}$, using a x20 objective and 535/590 excitation filters.

Protein isolation and western blotting. Total cell lysates (RPE1, MC3T3) were isolated using RIPA buffer supplemented with 1:100 protease (Applied Biological Materials, G135). Mouse tissue disruption and homogenization was performed in RIPA buffer supplemented with 1:100 protease using Tissue Lyser LT (Qiagen), for $5 \mathrm{~min}$ at $50 \mathrm{~Hz}$. Protein concentrations were determined using Bradford reagent (Bio-Rad). Lysates were boiled at $95{ }^{\circ} \mathrm{C}$ for $5 \mathrm{~min}$ and separated by SDS-PAGE electrophoresis using 8-16\% Tris-Glycine gels (Invitrogen) at $225 \mathrm{~V}$ for $30 \mathrm{~min}$. Samples were then transferred onto nitrocellulose membranes using an iBlot transfer system (Invitrogen) for $5 \mathrm{~min}$, and blocked using 5\% w/v nonfat dry milk dissolved in TBST (1X TBS, 0.05\% Tween-20) for $1 \mathrm{~h}$ at room temperature. Membranes were then incubated with the following primary antibodies: rabbit anti-Profilin 1 (Novus Biologicals, NB200-262, 1:10’000 for human lysates; Invitrogen, PA517444, 1:5'000 for murine lysates); mouse anti-p53 (Cell Signaling, 2524, 1:1'000); mouse anti- $\alpha$ Tubulin (Sigma, T6074; 1:15'000). Membranes were then incubated with secondary antibodies conjugated with HRP for $1 \mathrm{~h}$ at room temperature. The bands were visualised using enhanced chemiluminescence detection reagents (Advansta) and autoradiographic films (Aurogene). Equal loading was confirmed by using antibody against $\alpha$-Tubulin. The intensity of the western blot signals was determined by densitometry analysis using the ImageJ software and normalised to the density value of the loading control ( $\alpha$-Tubulin).

Indirect immunofluorescence. Cells were grown on coverslips, washed in 1x PBS and fixed in 4\% paraformaldehyde for $15 \mathrm{~min}$ at room temperature. Coverslips were blocked in 4\% FBS in 1x PBS $+0.1 \%$ Triton X-100 prior to incubation with primary antibodies diluted in blocking buffer: rabbit anti- $\gamma$-H2AX (Cell Signaling, 2577, 1:400); mouse anti-p53 (Cell Signaling, 2524, 1:2'000); mouse anti- $\alpha$-Tubulin (Sigma, T6074; 1:500); rabbit anti-Profilin 1 (Novus Biologicals, NB200-262, 
1:100); mouse anti-Aurora B (BD Transduction Laboratories, $611082,1: 100)$ overnight at $4{ }^{\circ} \mathrm{C}$. Samples were washed 3 times in 1x PBS and primary antibodies were detected using speciesspecific fluorescent secondary antibodies (Life Technologies). Samples were washed 3 more times in 1x PBS before DNA detection with $1 \mu \mathrm{g} / \mathrm{ml}$ Hoechst 33342 (Invitrogen, H3570) for 15 min at room temperature. Cells were stained with $5 \mu \mathrm{M}$ rhodamine phalloidin (Sigma, P1951) for 15 min at room temperature to visualise F-actin. Coverslips were washed 2 times in 1X PBS and then in distilled water and mounted with Mowiol. The cells were then examined using a Nikon's A1R confocal laser microscope. NIS-Elements Advanced Research Analysis software was used to quantify phalloidin fluorescence intensity.

Haematoxylin and eosin staining. Haematoxylin and eosin staining was conducted on paraffinembedded tissue. All sections used for histological analysis were 5- $\mu \mathrm{m}$ thick. Sections were deparaffinised using xylene for $30 \mathrm{~min}$ and rehydrated through a graded series of alcohol $(100,90$, 80 and $70 \%$ alcohol, each for $5 \mathrm{~min}$ ). The slices were then incubated with haematoxylin solution (Sigma Aldrich) for 1 minute and, after rinsing in distilled water, with eosin for 3 minutes. Afterwards, the sections were dehydrated in ethyl alcohol solution of gradient concentrations, embedded in the mounting medium, covered with a coverslip and analysed by transmission light microscopy (Nikon Intensilight C-HGFI).

Scoring of nuclear abnormalities. Mitotic defects (misaligned or lagging chromosomes, multipolar metaphases, anaphase bridges) were counted manually from Hoechst-stained CRISPR RPE1 cells visualised at Nikon's A1R confocal laser microscope. Experiments were repeated three times and on average 100 cells were counted for each experiment. Anaphase bridges and lagging chromosomes in shRNA RPE1 were counted in two independent experiment sets and $\geq 30$ mitoses were analysed in each experiment. Micronucleated cells, binucleated cells, and cells with chromosome bridges were counted manually from Hoechst-stained MC3T3 visualised at Nikon's A1R confocal laser microscope. Criteria for micronuclei scoring were as follows: i) diameter of micronuclei less than one-third of the nucleus; ii) intensity of Hoechst-stained micronuclei similar to that of the main nucleus; iii) micronuclear boundary distinguishable from the nuclear. Experiments were repeated at least 5 times and at least 100 cells were counted for each experiment. Due to similar scoring, results from the two $P f n 1^{+/}$cell clones (\#15 and \#51) were grouped into one data set. Micronucleation and binucleation in primary mesenchymal cells derived from WT and knock-in mice were assessed manually from Hoechst-positive cells. At least four biological 
replicates per genotype were analysed and about 100-200 cells were counted for each experiment. Binucleated cells within the femoral sections were scored using 3 biological replicates per genotype; at least five slices per sample were analysed and on average 50 cells per field were counted.

Genomic DNA extraction. Genomic DNA from OS/PDB tissues and matched healthy bone marrow samples was isolated using the High Pure PCR Template Preparation Kit following the manufacturer's instructions (Roche Life Science). Genomic DNA from cell lines (MC3T3 and RPE1) was extracted using the Proteinase K Method as previously described ${ }^{26}$. For WES samples, target enrichment was performed using the Agilent SureSelect Human All Exon V6 design (Agilent, Santa Clara, CA, USA).

Whole-genome and whole-exome sequencing of OS from PDB patients. Whole-genome and exome libraries were sequenced on the HiSeq 4000 platform (Illumina) in 151 bp paired-end mode. Raw sequencing reads were mapped to the GRCh38 build of the human reference genome using BWA-MEM version 0.7.17-r1188 [Ref. ${ }^{80}$ ]. Aligned reads in BAM format were processed following the Genome Analysis Toolkit (GATK, version 4.1.8.0) Best Practices workflow to remove duplicates and recalibrate base quality scores ${ }^{81}$. Somatic point mutations and indels were detected using Mutect ${ }^{82}$ (GATK, version 4.1.8.0), MuSE $^{83}$ (version 1.0rc) and Strelka2 [Ref. ${ }^{84}$ ] (version 2.9.2) using the matching germline data as control. Each algorithm was run independently on each tumour-normal pair, and the calls were integrated using the Python library mergevcf (https://github.com/ljdursi/mergevef). Only mutations detected by at least $2 / 3$ algorithms were considered for further analysis. For the WGS data, somatic copy-number aberrations, ploidy and purity values were detected using ascatNGS ${ }^{85}$. Structural variants were detected using Delly ${ }^{86}$ (version 0.8.3), LUMPY ${ }^{87}$ (version 9-120.2.13), Manta $^{88}$ (version 1.6.0), and SvABA ${ }^{89}$ (version 1.1.3). Each tool was run independently on each tumour-normal pair. The calls generated by each algorithm were merged using mergevef, and only structural variants detected by at least two algorithms were considered for further analysis. For WES samples, somatic copy-number aberrations were detected by integrating the output of GATK (version 4.1.8.0) and FreeBayes ${ }^{90}$ (version 1.3) using PureCN (https://github.com/lima1/PureCN). Briefly, the GATK4 Somatic CNV workflow (GATK, version 4.1.8.0) was utilized for the normalization of read counts and genome segmentation (using 2 unrelated normal samples as controls). FreeBayes was used to obtain B-allele frequency values for gnomAD (version 3.1) variant sites with population allele frequencies greater 
than $0.1 \%$. Finally, PureCN was used to integrate the output of GATK and FreeBayes to estimate the allele-specific consensus copy-number profile, purity and ploidy for each tumour sample. For both WES and WGS samples, we considered that a tumour underwent one WGD if the copynumber of the major allele (i.e., the most amplified allele) was equal or greater than 2 in $>=50 \%$ of the genome. Timing of WGD events was performed as previously described ${ }^{58,91}$. The basic idea is that mutations occurring before a genome doubling would be present in multiple copies, whereas mutations occurring after WGD would be likely present in just one copy. Thus, the ratio of early versus late mutations informs about the timing of WGD during tumour evolution.

Whole-genome sequencing of $\boldsymbol{P} \boldsymbol{f n} \boldsymbol{I}^{+/-}$MC3T3 clones. Low-pass whole-genome sequencing ( 7x) of the different Pfnl knock-out clones and WT MC3T3 cells was performed on a HiSeq 4000 (Illumina) to obtain $100 \mathrm{bp}$ paired-end reads. Raw sequencing reads were mapped to the GRCm38 build of the mouse reference genome using BWA-MEM version 0.7.17-r1188. Aligned reads in BAM format were processed following the Genome Analysis Toolkit (GATK, version 4.1.8.0) Best Practices workflow. Since the ploidy of the WT clone is $\sim 4 \mathrm{n}$, we also downloaded whole-genome data from the C57BL_6NJ line (ftp://ftp-mouse.sanger.ac.uk/current_bams/C57BL_6NJ.bam) to be used as a reference sample for copy-number analysis rather than the WT clone. The C57BL_6NJ data were downsampled to achieve a similar depth of coverage as the MC3T3 clones. Copy-number analysis was performed using $\mathrm{CNVkit}^{92}$ (version 0.9.9). CNVkit was run using a window size of 50 Kbp and a ploidy of 4. Finally, chromosome-level copy number values were computed as the modal copy number for each chromosome normalised by segment size.

Statistical Analysis. All statistical analyses were performed using GraphPad Prism 6.0. All results are presented in graphs as the mean \pm standard error of the mean (s.e.m.). Data were obtained from at least three independent experiments (unless otherwise noted in the figure legends), and have been overlaid with dot plots showing the values of each experiment. Each exact $n$ value is indicated in the corresponding figure legend. Western blot data presented are representative of at least three independent experiments that yielded similar results, unless otherwise noted in the figure legends. Immunofluorescence staining experiments were independently repeated at least three times; all the confocal images shown are representative of a minimum of 15 images. Student's $t$-test was used for comparing the statistical significance between 2 groups and all tests were determined using unpaired one-sided tests. Comparisons between multiple groups were assessed by one-way analysis of variance (ANOVA) with Dunnet's multiple comparisons test or two-way ANOVA with Bonferroni's or Sidak's multiple comparisons test. Differences between $P F N 1^{+/-}$and $P F N 1^{-/-}$RPE1 
cells were statistically significant only when specified in the graphs. Specific $P$ values are labelled in the figure legends, where significant values are $P<0.05$. No data were excluded from the analyses presented in this study. 
Acknowledgments. We are grateful to members of the Integrated Microscopy, Mouse Modelling, and FACS Facilities of IGB-CNR (Naples, Italy). We also thank Dr Annalisa Fico (Institute of Genetics and Biophysics, National Research Council of Italy, Naples) for her help and advice in the lentiviral infection of RPE1 cells. Authors are also grateful to Alessandro Sacco and Dr Giuseppe Viglietto (Department of Experimental and Clinical Medicine, University Magna Græcia of Catanzaro, Italy) for their support during fluorescence time-lapse experiments. We thank the EuroBioImaging facility at Institute for Experimental Endocrinology and Oncology (CNR), Naples, Italy for help with microscopy experiments. The research leading to these results has received funding from AIRC under IG 2020 - ID. 25110 project - P.I. F.G. F.S.d.C. was supported by Fondazione Umberto Veronesi. F.M. and I.C.-C. acknowledge funding from EMBL.

Author contribution. F.S.d.C. and F.G. conceived the study and wrote the paper with input from F.M., and I.C.-C. F.S.d.C., F.G., F.M., and I.C.-C. drafted the figures and tables. F.S.d.C. carried out in vitro and ex vivo experiments, and performed statistical analysis. S.R. performed histological analysis on mouse sections. F.S.d.C., S.R., and F.G. analysed the results. F.M. and I.C.-C. performed analyses of sequencing data. M.M., A.C.D.L., and F.B. helped in time-lapse imaging and provided microscopy infrastructures. L.P and K.S. obtained clinical specimens and provided clinical information. I.C.-C. contributed to discussion. F.G. supervised the study and data analysis. All authors read and approved the final version of the manuscript.

Competing interests. The authors declare no competing interests.

Data availability. All relevant data supporting the key findings of this study are available within the article. Raw sequencing data are available upon request. 


\section{References}

1. Levine, M. S. \& Holland, A. J. The impact of mitotic errors on cell proliferation and tumorigenesis. Genes Dev. 32, 620-638 (2018).

2. Jallepalli, P. V. \& Lengauer, C. Chromosome segregation and cancer: cutting through the mystery. Nat. Rev. Cancer 1, 109-117 (2001).

3. Ramkumar, N. \& Baum, B. Coupling changes in cell shape to chromosome segregation. Nat. Rev. Mol. Cell Biol. 17, 511-521 (2016).

4. Kops, G. J. P. L., Weaver, B. A. A. \& Cleveland, D. W. On the road to cancer: aneuploidy and the mitotic checkpoint. Nat. Rev. Cancer 5, 773-785 (2005).

5. Naylor, R. M. \& Van Deursen, J. M. Aneuploidy in Cancer and Aging. Annu. Rev. Genet. 50, 45-66 (2016).

6. Potapova, T. A., Zhu, J. \& Li, R. Aneuploidy and chromosomal instability: A vicious cycle driving cellular evolution and cancer genome chaos. Cancer Metastasis Rev. 32, 377-389 (2013).

7. Santaguida, S. \& Amon, A. Short- and long-term effects of chromosome mis-segregation and aneuploidy. Nat. Rev. Mol. Cell Biol. 16, 473-485 (2015).

8. Sansregret, L., Vanhaesebroeck, B. \& Swanton, C. Determinants and clinical implications of chromosomal instability in cancer. Nat Rev Clin Oncol 15, 139-150 (2018).

9. McClintock, B. The Behavior in Successive Nuclear Divisions of a Chromosome Broken at Meiosis. Proc Natl Acad Sci U S A 25, 405-416 (1939).

10. McClintock, B. The Stability of Boken Ends of Chromosomes in Zea Mays. Genetics 26, 234-82 (1941).

11. Campbell, P. J. et al. The patterns and dynamics of genomic instability in metastatic pancreatic cancer. Nature 467, 1109-1113 (2010).

12. Liu, S. et al. Nuclear envelope assembly defects link mitotic errors to chromothripsis. Nature 561, 551-555 (2018). 
13. Aguilera, A. \& García-Muse, T. Causes of genome instability. Annu. Rev. Genet. 47, 1-32 (2013).

14. Beroukhim, R. et al. The landscape of somatic copy-number alteration across human cancers. Nature 463, 899-905 (2010).

15. Cortés-Ciriano, I. et al. Comprehensive analysis of chromothripsis in 2,658 human cancers using whole-genome sequencing. Nat. Genet. 52, 331-341 (2020).

16. Mirabello, L., Troisi, R. J. \& Savage, S. A. Osteosarcoma incidence and survival rates from 1973 to 2004: Data from the surveillance, epidemiology, and end results program. Cancer 115, 1531-1543 (2009).

17. Chen, X. et al. Recurrent somatic structural variations contribute to tumorigenesis in pediatric osteosarcoma. Cell Rep. 7, 104-12 (2014).

18. Behjati, S. et al. Recurrent mutation of IGF signalling genes and distinct patterns of genomic rearrangement in osteosarcoma. Nat. Commun. 8, 15936 (2017).

19. Gianferante, D. M., Mirabello, L. \& Savage, S. A. Germline and somatic genetics of osteosarcoma - Connecting aetiology, biology and therapy. Nat Rev Endocrinol. 13, 480-491 (2017).

20. Siegel, R. L., Miller, K. D. \& Jemal, A. Cancer statistics, 2019. CA. Cancer J. Clin. 69, 7-34 (2019).

21. McCarthy, E. F. Bone tumors. in Rheumatology: Sixth Edition 1734-1743 (2015).

22. Longhi, A., Errani, C., Gonzales-Arabio, D., Ferrari, C. \& Mercuri, M. Osteosarcoma in patients older than 65 years. J. Clin. Oncol. 26, 5368-73 (2008).

23. Huvos, A. G. Osteogenic sarcoma of bones and soft tissues in older persons. A clinicopathologic analysis of 117 patients older than 60 years. Cancer 57, 1442-1449 (1986).

24. Rickel, K., Fang, F. \& Tao, J. Molecular genetics of osteosarcoma. Bone 102, 69-79 (2017).

25. Perry, J. A. et al. Complementary genomic approaches highlight the PI3K/mTOR pathway as a common vulnerability in osteosarcoma. Proc. Natl. Acad. Sci. U. S. A. 111, 5564-5573 
(2014).

26. Scotto di Carlo, F., Pazzaglia, L., Esposito, T. \& Gianfrancesco, F. The loss of Profilin 1 causes early-onset Paget's disease of bone. J. Bone Miner. Res. 35, 1387-1398 (2020).

27. Witke, W. The role of profilin complexes in cell motility and other cellular processes. Trends in Cell Biology 14, 461-469 (2004).

28. Witke, W. et al. In mouse brain profilin I and profilin II associate with regulators of the endocytic pathway and actin assembly. EMBO J. 17, 967-976 (1998).

29. Pimm, M. L., Hotaling, J. \& Henty-Ridilla, J. L. Profilin choreographs actin and microtubules in cells and cancer. Int Rev Cell Mol Biol. 355, 155-204 (2020).

30. Witke, W., Sutherland, J. D., Sharpe, A., Arai, M. \& Kwiatkowski, D. J. Profilin I is essential for cell survival and cell division in early mouse development. Proc. Natl. Acad. Sci. U. S. A. 98, 3832-6 (2001).

31. Janke, J. et al. Suppression of tumorigenicity in breast cancer cells by the microfilament protein profilin 1. J. Exp. Med. 191, 1675-1685 (2000).

32. Zou, L. et al. Profilin-1 is a negative regulator of mammary carcinoma aggressiveness. Br. J. Cancer 97, 1361-1371 (2007).

33. Ding, Z. et al. Profilin-1 downregulation has contrasting effects on early vs late steps of breast cancer metastasis. Oncogene 33, 2065-2074 (2014).

34. Wu, N. et al. Profilin 1 obtained by proteomic analysis in a 11-trans retinoic acid-treated hepatocarcinoma cell lines is involved in inhibition of cell proliferation and migration. Proteomics 6, 6095-6106 (2006).

35. Grønborg, M. et al. Biomarker discovery from pancreatic cancer secretome using a differential proteomic approach. Mol Cell Proteomics 5, 157-171 (2006).

36. Yao, W. et al. Profilin-1 suppresses tumorigenicity in pancreatic cancer through regulation of the SIRT3-HIF1 $\alpha$ axis. Mol. Cancer 13, 1-12 (2014).

37. Zoidakis, J. et al. Profilin 1 is a potential biomarker for bladder cancer aggressiveness. Mol. 
Cell. Proteomics 11, 1-15 (2012).

38. Giansanti, M. G. et al. Cooperative interactions between the central spindle and the contractile ring during Drosophila cytokinesis. Genes Dev. 12, 396-410 (1998).

39. Böttcher, R. T. et al. Profilin 1 is required for abscission during late cytokinesis of chondrocytes. EMBO J. 28, 1157-1169 (2009).

40. Taubenberger, A. V., Baum, B. \& Matthews, H. K. The Mechanics of Mitotic Cell Rounding. Front. cell Dev. Biol. 8, 687 (2020).

41. Bakhoum, S. F. et al. Numerical chromosomal instability mediates susceptibility to radiation treatment. Nat. Commun. 6, 5990 (2015).

42. Passerini, V. et al. The presence of extra chromosomes leads to genomic instability. Nat. Commun. 7, 10754 (2016).

43. Wilhelm, T. et al. Mild replication stress causes chromosome mis-segregation via premature centriole disengagement. Nat. Commun. 10, 3585 (2019).

44. Harasymiw, L. A., Tank, D., McClellan, M., Panigrahy, N. \& Gardner, M. K. Centromere mechanical maturation during mammalian cell mitosis. Nat. Commun. 10, 1761 (2019).

45. Stojic, L. et al. A high-content RNAi screen reveals multiple roles for long noncoding RNAs in cell division. Nat. Commun. 11, 1851 (2020).

46. McCollum, D. Cytokinesis: the central spindle takes center stage. Curr. Biol. 14, 953-955 (2004).

47. Albertson, R., Cao, J., Hsieh, T. S. \& Sullivan, W. Vesicles and actin are targeted to the cleavage furrow via furrow microtubules and the central spindle. J. Cell Biol. 181, 777-790 (2008).

48. Maddox, A. S. \& Burridge, K. RhoA is required for cortical retraction and rigidity during mitotic cell rounding. J. Cell Biol. 160, 255-265 (2003).

49. Toyoda, Y. et al. Genome-scale single-cell mechanical phenotyping reveals disease-related genes involved in mitotic rounding. Nat. Commun. 8, 1266 (2017). 
50. ML, L. et al. Chromothripsis as an on-target consequence of CRISPR-Cas9 genome editing. Nat. Genet. 53, 895-905 (2021).

51. Papathanasiou, S. et al. Whole chromosome loss and genomic instability in mouse embryos after CRISPR-Cas9 genome editing. Nat. Commun. 12, 5855 (2021).

52. Tommasi, S., Besaratinia, A., Wilczynski, S. P. \& Pfeifer, G. P. Loss of Rassf1a enhances p53-mediated tumor predisposition and accelerates progression to aneuploidy. Oncogene 30, 690-700 (2010).

53. Donehower, L. A. et al. Mice deficient for p53 are developmentally normal but susceptible to spontaneous tumours. Nature 356, 215-221 (1992).

54. Fukasawa, K. \& Vande Woude, G. F. Synergy between the Mos/mitogen-activated protein kinase pathway and loss of p53 function in transformation and chromosome instability. Mol. Cell. Biol. 17, 506-518 (1997).

55. Duensing, A. \& Duensing, S. Guilt by association? p53 and the development of aneuploidy in cancer. Biochem. Biophys. Res. Commun. 331, 694-700 (2005).

56. Haupt, Y., Maya, R., Kazaz, A. \& Oren, M. Mdm2 promotes the rapid degradation of p53. Nature 387, 296-299 (1997).

57. Kastenhuber, E. R. \& Lowe, S. W. Putting p53 in Context. Cell 170, 1062-1078 (2017).

58. Steele, C. D. et al. Undifferentiated Sarcomas Develop through Distinct Evolutionary Pathways. Cancer Cell 35, 441-456 (2019).

59. Gerstung, M. et al. The evolutionary history of 2,658 cancers. Nature 578, 122-128 (2020).

60. Kunda, P., Pelling, A. E., Liu, T. \& Baum, B. Moesin controls cortical rigidity, cell rounding, and spindle morphogenesis during mitosis. Curr. Biol. 18, 91-101 (2008).

61. Kunda, P. \& Baum, B. The actin cytoskeleton in spindle assembly and positioning. Trends Cell Biol. 19, 174-179 (2009).

62. Luxenburg, C., Amalia Pasolli, H., Williams, S. E. \& Fuchs, E. Developmental roles for Srf, cortical cytoskeleton and cell shape in epidermal spindle orientation. Nat. Cell Biol. 13, 203- 
$214(2011)$.

63. Nishimura, K. et al. Cdk1-mediated DIAPH1 phosphorylation maintains metaphase cortical tension and inactivates the spindle assembly checkpoint at anaphase. Nat. Commun. 10, 981 (2019).

64. Wei, Z. et al. Mutations in Profilin 1 Cause Early-Onset Paget's Disease of Bone With Giant Cell Tumors. J. Bone Miner. Res. 36, 1088-1103 (2021).

65. Mardin, B. R. et al. A cell-based model system links chromothripsis with hyperploidy. Mol. Syst. Biol. 11, 828 (2015).

66. Drainas, A. P. et al. Genome-wide Screens Implicate Loss of Cullin Ring Ligase 3 in Persistent Proliferation and Genome Instability in TP53-Deficient Cells. Cell Rep. 31, $107465(2020)$.

67. Umbreit, N. T. et al. Mechanisms generating cancer genome complexity from a single cell division error. Science 368, eaba0712 (2020).

68. Weaver, B. A. A., Silk, A. D., Montagna, C., Verdier-Pinard, P. \& Cleveland, D. W. Aneuploidy Acts Both Oncogenically and as a Tumor Suppressor. Cancer Cell 11, 25-36 (2007).

69. Dobles, M., Liberal, V., Scott, M. L., Benezra, R. \& Sorger, P. K. Chromosome missegregation and apoptosis in mice lacking the mitotic checkpoint protein Mad2. Cell 101, 635-645 (2000).

70. Iwanaga, Y. et al. Heterozygous deletion of mitotic arrest-deficient protein 1 (MAD1) increases the incidence of tumors in mice. Cancer Res. 67, 160-166 (2007).

71. Ko, M. A. et al. Plk4 haploinsufficiency causes mitotic infidelity and carcinogenesis. Nat. Genet. 37, 883-888 (2005).

72. Jeganathan, K., Malureanu, L., Baker, D. J., Abraham, S. C. \& Van Deursen, J. M. Bub1 mediates cell death in response to chromosome missegregation and acts to suppress spontaneous tumorigenesis. J. Cell Biol. 179, 255-267 (2007). 
73. Zasadil, L. M., Britigan, E. M. C. \& Weaver, B. A. 2n or not 2n: Aneuploidy, polyploidy and chromosomal instability in primary and tumor cells. Semin Cell Dev Biol. 24, 370-379 (2013).

74. Thompson, S. L. \& Compton, D. A. Proliferation of aneuploid human cells is limited by a p53-dependent mechanism. J. Cell Biol. 188, 369-381 (2010).

75. Fujiwara, T. et al. Cytokinesis failure generating tetraploids promotes tumorigenesis in p53null cells. Nature 437, 1043-1047 (2005).

76. Lens, S. M. A. \& Medema, R. H. Cytokinesis defects and cancer. Nat. Rev. Cancer 19, 3245 (2019).

77. Zhang, C. Z. et al. Chromothripsis from DNA damage in micronuclei. Nature 522, 179-184 (2015).

78. Maciejowski, J. et al. APOBEC3-dependent kataegis and TREX1-driven chromothripsis during telomere crisis. Nat. Genet. 52, 884-890 (2020).

79. Fan, Y. et al. Profilin-1 phosphorylation directs angiocrine expression and glioblastoma progression through HIF-1 $\alpha$ accumulation. Nat. Cell Biol. 16, 445-456 (2014).

80. Li, H. \& Durbin, R. Fast and accurate short read alignment with Burrows-Wheeler transform. Bioinformatics 25, 1754-1760 (2009).

81. Van der Auwera, G. A. et al. From FastQ data to high confidence variant calls: the Genome Analysis Toolkit best practices pipeline. Curr. Protoc. Bioinforma. 43, 1-33 (2013).

82. Cibulskis, K. et al. Sensitive detection of somatic point mutations in impure and heterogeneous cancer samples. Nat. Biotechnol. 31, 213-219 (2013).

83. Fan, Y. et al. MuSE: accounting for tumor heterogeneity using a sample-specific error model improves sensitivity and specificity in mutation calling from sequencing data. Genome Biol. 17, 178 (2016).

84. Kim, S. et al. Strelka2: fast and accurate calling of germline and somatic variants. Nat. Methods 15, 591-594 (2018). 
85. Raine, K. M. et al. ascatNgs: Identifying Somatically Acquired Copy-Number Alterations from Whole-Genome Sequencing Data. Curr. Protoc. Bioinforma. 56, 1-17 (2016).

86. Rausch, T. et al. DELLY: structural variant discovery by integrated paired-end and split-read analysis. Bioinformatics 28, 333-339 (2012).

87. Layer, R. M., Chiang, C., Quinlan, A. R. \& Hall, I. M. LUMPY: a probabilistic framework for structural variant discovery. Genome Biol. 15, 84 (2014).

88. Chen, X. et al. Manta: rapid detection of structural variants and indels for germline and cancer sequencing applications. Bioinformatics 32, 1220-1222 (2016).

89. Wala, J. A. et al. SvABA: genome-wide detection of structural variants and indels by local assembly. Genome Res. 28, 581-591 (2018).

90. Garrison, E. \& Marth, G. Haplotype-based variant detection from short-read sequencing. arXiv:1207.3907 (2012).

91. Campbell, P. J. et al. Pan-cancer analysis of whole genomes. Nature 578, 82-93 (2020).

92. Talevich, E., Shain, A. H., Botton, T. \& Bastian, B. C. CNVkit: Genome-Wide Copy Number Detection and Visualization from Targeted DNA Sequencing. PLOS Comput. Biol. 12, $1004873(2016)$. 
Figure 1: Profilin 1 localisation during the mitotic stages. Immunofluorescent staining for the mitotic spindle ( $\alpha$-tubulin, magenta) and Profilin 1 (green) in normal RPE1 cells during mitosis; DNA is shown in blue (Hoechst 33342). Panels on the left represent the merge of the different channels; scale bars $5 \mu \mathrm{m}$. 
Figure 2
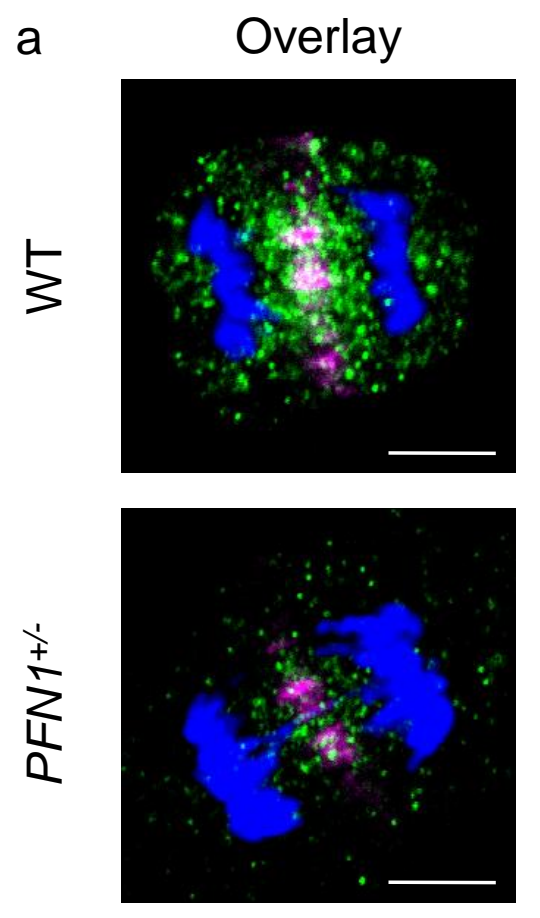

Hoechst

Aurora B

Profilin 1
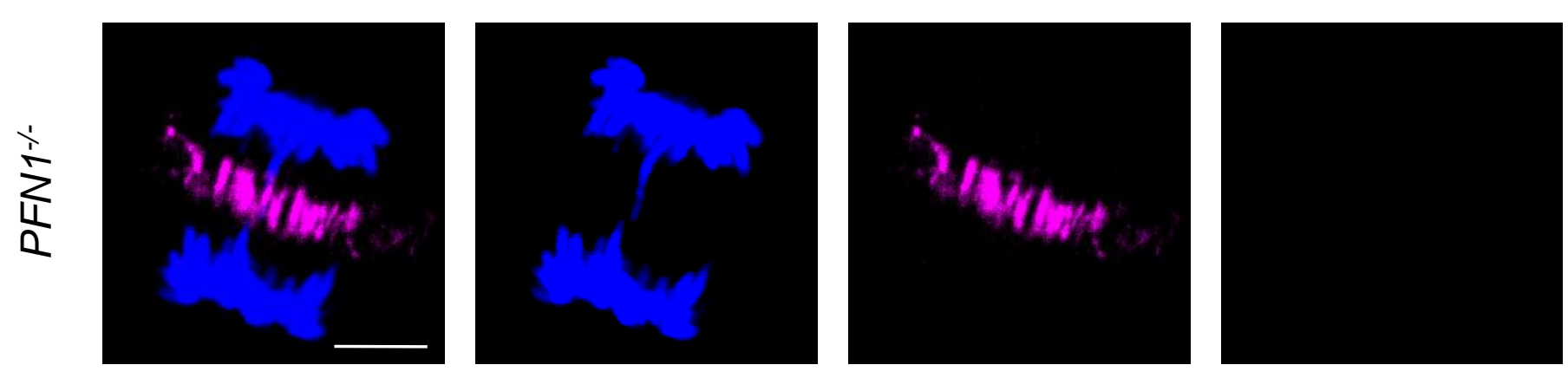

b

WT

PFN1+/-

PFN1/-

C
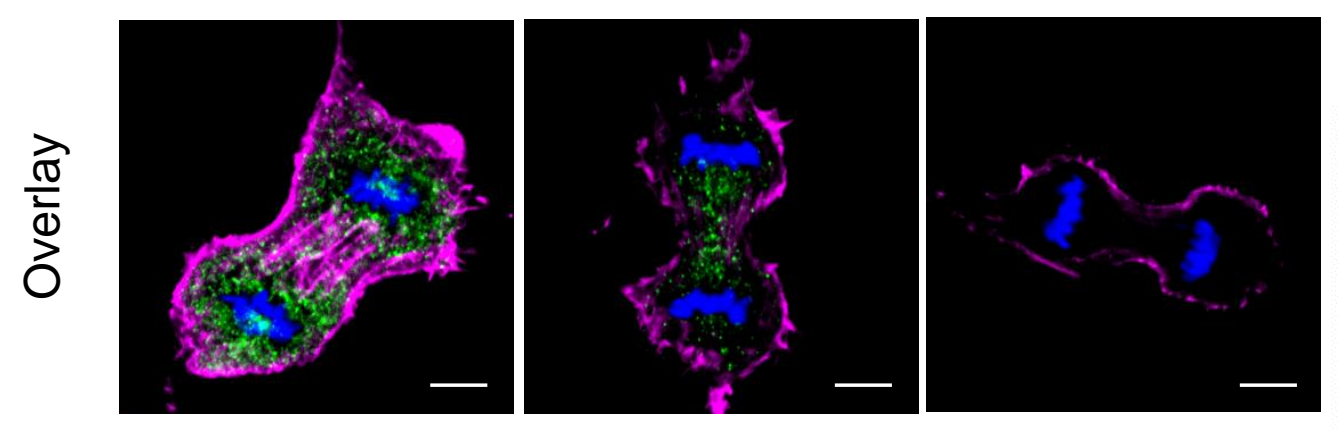

$\frac{}{\frac{}{0}}$
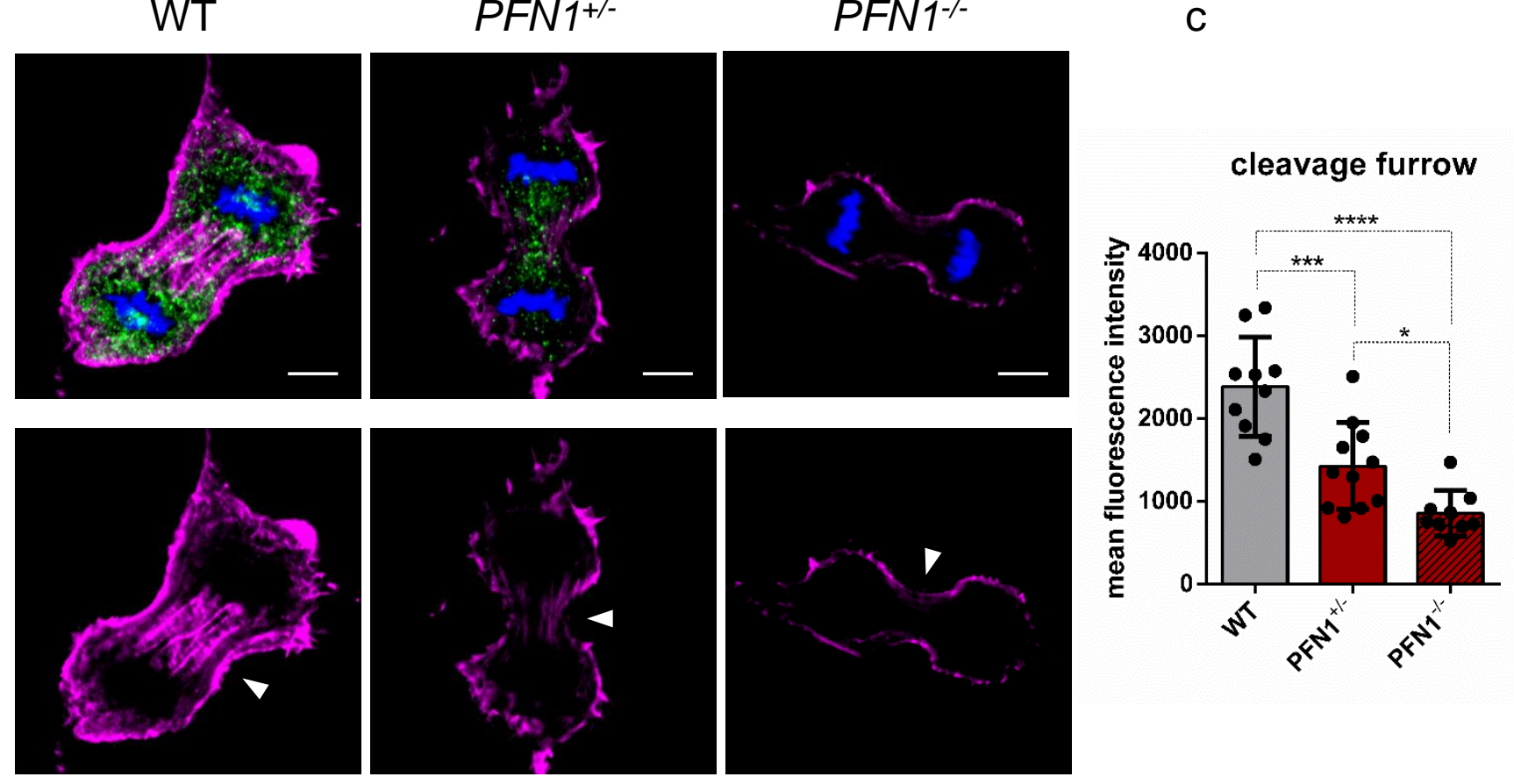

Phalloidin/Hoechst/Profilin 1 
Figure 2: Profilin 1 localises in the mitotic midzone and promotes actin polymerisation at the cleavage furrow. (a) $\mathrm{WT}, P F N 1^{+/-}$and $P F N 1^{-/-}$RPE1 cells at anaphase stained for DNA (Hoechst 33342, blue), a midzone marker (Aurora B kinase, magenta) and Profilin 1 (green). Panels on the left represent the merge of the different channels; scale bars $5 \mu \mathrm{m}$. Note that PFN1-KO cells show normal Aurora B localisation. (b) WT, $P F N 1^{+/}$and $P F N 1^{-/-}$RPE1 cells at telophase stained for DNA (Hoechst 33342, blue), Profilin 1 (green) and F-actin (phalloidin, magenta). Panels on the top represent the merge of the different channels. Arrowheads point towards furrow ingression; scale bars $5 \mu \mathrm{m}$. (c) Mean fluorescence intensity of phallodin staining at the cleavage furrow; data are shown as mean \pm s.e.m.; dots represent the fluorescent intensity for each sample $(n=10$ for WT, 11 for $P F N 1^{+/}, 9$ for $P F N 1^{-/}$cells $) ; * * P=0.0004, * * * * P<0.0001, * P=0.0461$. Ordinary one-way ANOVA performed. 
Figure 3: Profilin 1 knock-out induces prolonged mitosis. (a) schematic of the experiment. (b) Percentage of cells able to complete mitosis following the round up; data are shown as mean \pm s.e.m.; dots represent the mean for each experiment ( $n=67 \mathrm{WT}, 44 \mathrm{PFNI}^{+/-}, 43 \mathrm{PFN1}^{-/-} \mathrm{RPE} 1$ cells); $* * * P=0.0005, * * P=0.0023$. The mean difference between $P F N 1^{+/}$and $P F N 1^{-/}$cells was not statistically significant $(P=0.4310)$. (c) Time taken from start (cell rounding) to end (cytokinesis) of mitosis; data are shown as mean \pm s.e.m.; dots represent the duration of each cell division expressed in minutes $\left(n\right.$ mitoses $=39 \mathrm{WT} 8 \mathrm{PFN1}^{+/}, 14 P F N 1^{-/} \mathrm{RPE} 1$ cells $)$; $* * * * P<$ 0.0001. Data in b and $\mathbf{c}$ were analysed by ordinary one-way ANOVA. (d) Representative time-lapse phase-contrast images (taken every 2 minutes) of WT (top row) and PFN1-KO (bottom row) RPE1 cells; scale bars $25 \mu \mathrm{m}$. Time stamps indicate elapsed time in hours:minutes. (e) WT, $P F N 1^{+/-}$and $P F N 1^{-/}$RPE1 cells at metaphase stained for DNA (Hoechst 33342, blue), Profilin 1 (green) and Factin (phalloidin, magenta); scale bars $5 \mu \mathrm{m}$. (f) Mean fluorescence intensity of cortical actin; data are shown as mean \pm s.e.m.; dots represent the fluorescent intensity for each sample $(n=25$ for WT, 17 for $P F N 1^{+/}, 20$ for $P F N 1^{-/}$cells); ${ }^{* * *} P=0.0002, * * * * P<0.0001,{ }^{*} P=0.0436$. Ordinary oneway ANOVA performed. 


\section{Figure 4}

a
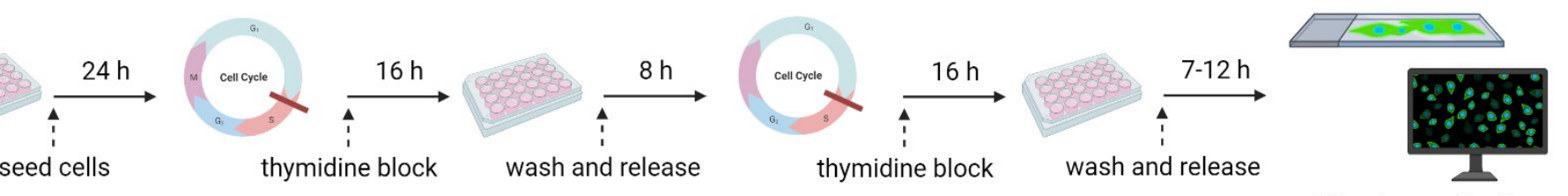

cell fixation and indirect immunofluorescence
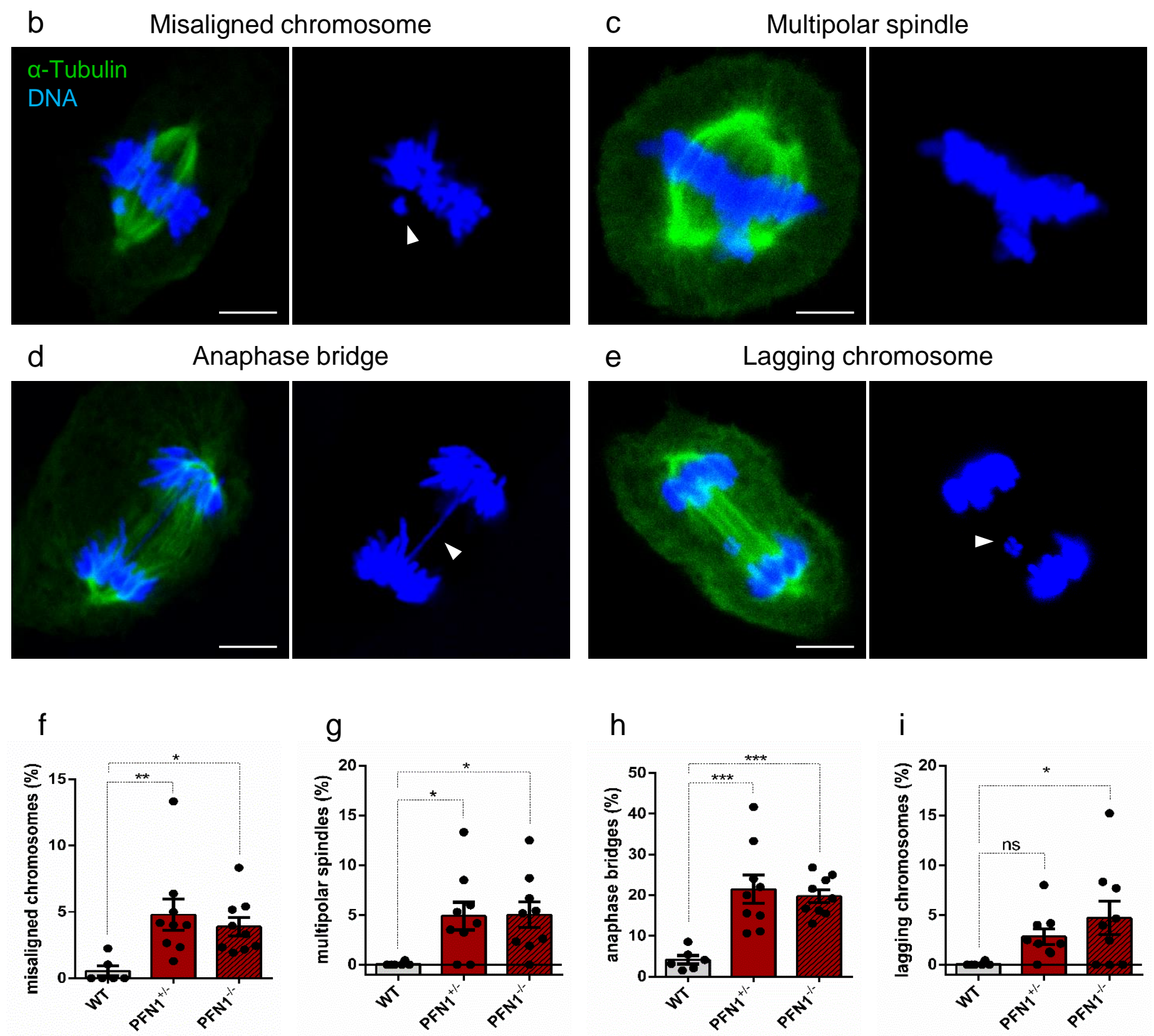
Figure 4: Chromosome segregation defects in Profilin 1-deficient RPE1 cells. (a) Schematic of the experiment. Below, representative immunofluorescence images of mitotic PFN1-KO RPE1 cells showing (b) chromosome misalignment (arrowhead) at the metaphase plate, (c) multipolar spindle formation, (d) a chromosome bridge (arrowhead) persisting in late anaphase, (e) telophase with a lagging chromosome (arrowhead). Cells were stained for microtubules ( $\alpha$-tubulin, green) and DNA (Hoechst 33342, blue); scale bars $5 \mu \mathrm{m}$. In each panel, the overlay ( $\alpha$-tubulin/DNA) is shown on the left. (f) Quantification (\%) of cells with misaligned chromosomes; ${ }^{*} P=0.0096,{ }^{*} P=0.0411$. (g) Quantification (\%) of cells with multipolar spindles; $* P=0.0305, * P=0.0259$. (h) Quantification (\%) of cells with anaphase bridges; $* * * P=0.0003$, $* * * P=0.0009$. (i) Quantification (\%) of cells with lagging chromosomes; ns $P=0.2457$, ${ }^{*} P=0.0345$. Data in $\mathbf{f - i}$ are shown as mean \pm s.e.m. of three independent experiments ( $n=2$ biological replicates for WT, 3 for $P F N 1^{+-}, 3$ for $P F N 1^{-/}$ cells); dots represent the value of each experiment (scoring 911, 825 and 616 total mitoses in WT, $\mathrm{PFNI}^{+/-}$and $\mathrm{PFNI}^{-/-}$samples, respectively). Ordinary one-way ANOVA performed. 
Figure 5: Profilin 1 deficiency is associated with nuclear abnormalities and DNA damage. Representative confocal images of $P f n 1^{+/-}$MC3T3 cells subjected to phalloidin (F-actin, red) and Hoechst 33342 (nucleus, blue) staining, showing nuclear abnormalities: (a) binucleated cells are indicated by arrowheads; (b) dashed lines indicate chromosome bridges connecting two cells; (c) micronuclei are indicated by white arrows; scale bars $10 \mu \mathrm{m}$. (d) Quantification (\%) of binucleated cells; data are shown as mean \pm s.e.m.; dots represent the value of each experiment $(n=508 \mathrm{WT}$ and $479 \mathrm{KO}$ cells); $* * * P=0.0004$. (e) Quantification (\%) of cells with chromosome bridges; data are shown as mean \pm s.e.m.; dots represent the value of each experiment $(n=800 \mathrm{WT}$ and $771 \mathrm{KO}$ cells); $* * P=0.0021$. (f) Quantification (\%) of micronucleated cells; data are shown as mean \pm s.e.m.; dots represent the value of each experiment ( $n=485 \mathrm{WT}$ and $496 \mathrm{KO}$ cells); $* * P=0.0049$. (g) Confocal image of a micronucleated $P f n 1^{+/}$MC3T3 cell stained for DNA (Hoechst 33342, blue) and $\gamma$-H2AX (green). Panel on the left represents the merge of the different channels; scale bar 5 $\mu \mathrm{m}$. (h) The mean percentage of cells positive to $\gamma-\mathrm{H} 2 \mathrm{AX}$ is shown as mean \pm s.e.m.; dots represent the value of each experiment ( $n=169 \mathrm{WT}$ and $324 \mathrm{KO}$ micronucleated cells); ${ }^{* * * *} P<0.0001$. Data in d,e,f,h were analysed by one-tailed unpaired Student's t-tests; results from $P f n 1^{+/-}$cell clones (\#15 and \#51) were grouped into one data set. (i) Western blotting analysis of WT and 2 different $P f n 1^{+/}$MC3T3 clones (\#15 and \#51) showing p53 accumulation. $\alpha$-Tubulin was used as loading control. (j) Quantification of p53 normalised to $\alpha$-Tubulin; bars represent mean \pm s.e.m.; dots represent the mean for each experiment ( $n=3$ from two independent protein extracts); $* * P=$ $0.0041,{ }^{* *} P=0.0023$. Ordinary one-way ANOVA test performed. (k) Confocal images of $\mathrm{p} 53$ nuclear localisation in WT and $P f n 1^{+/-}$clones (\#15 and \#51). DNA is stained with Hoechst 33342 (blue); scale bars $10 \mu \mathrm{m}$. 

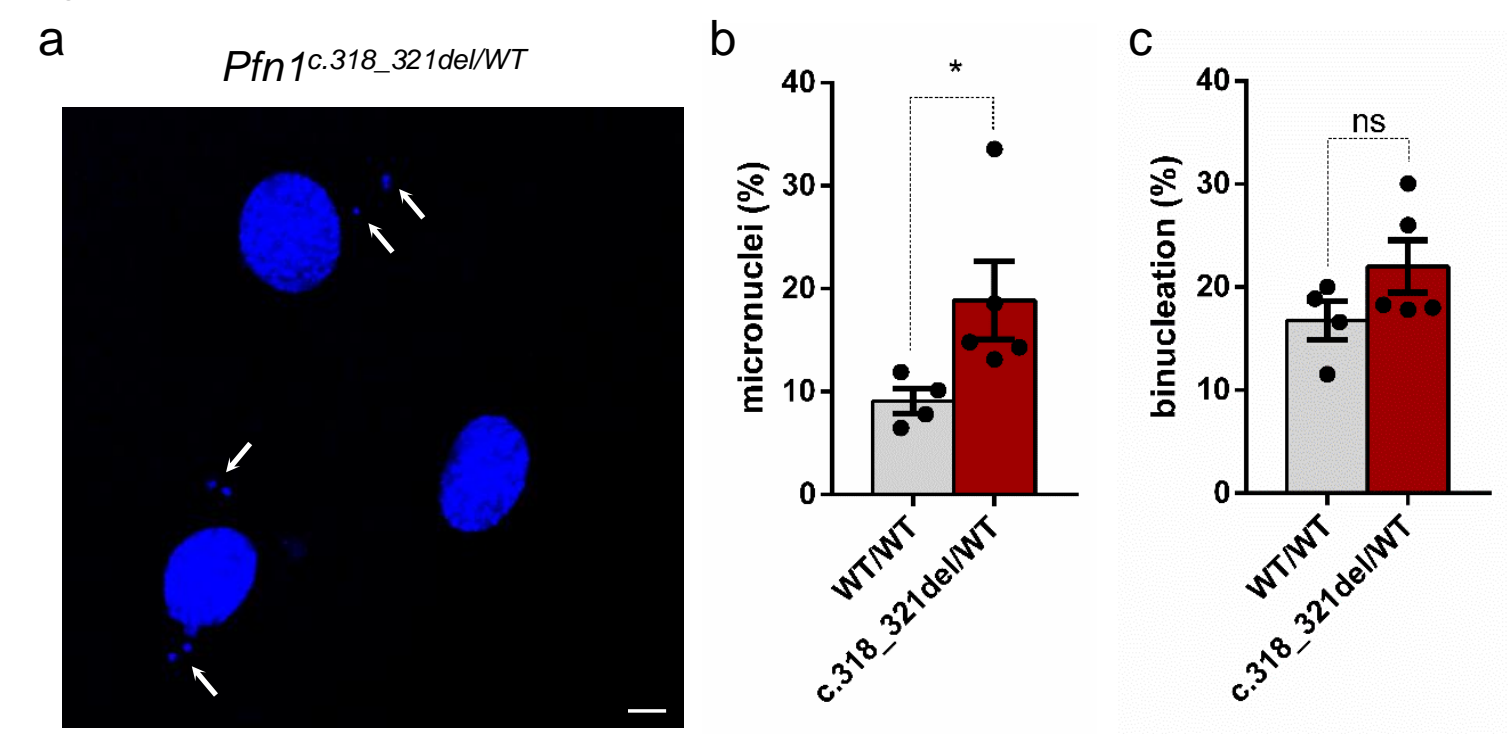

d

e

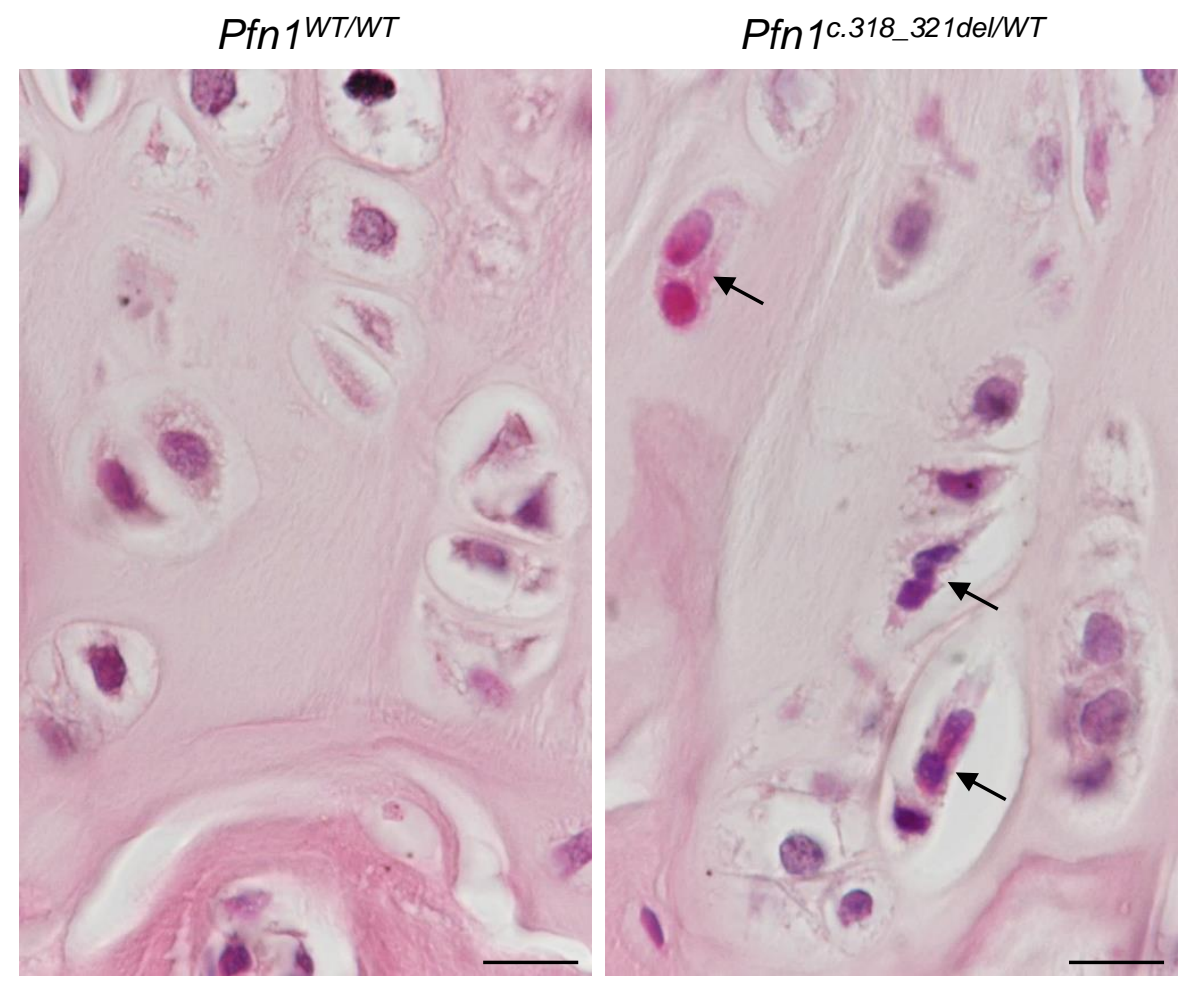

f

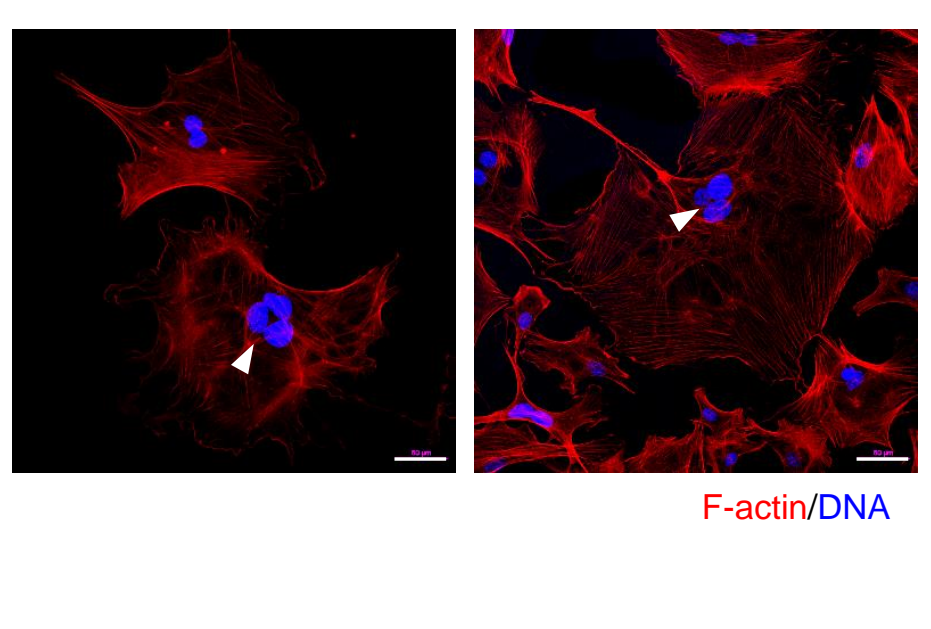

9 Pfn1c.318_321delWT

Histological section
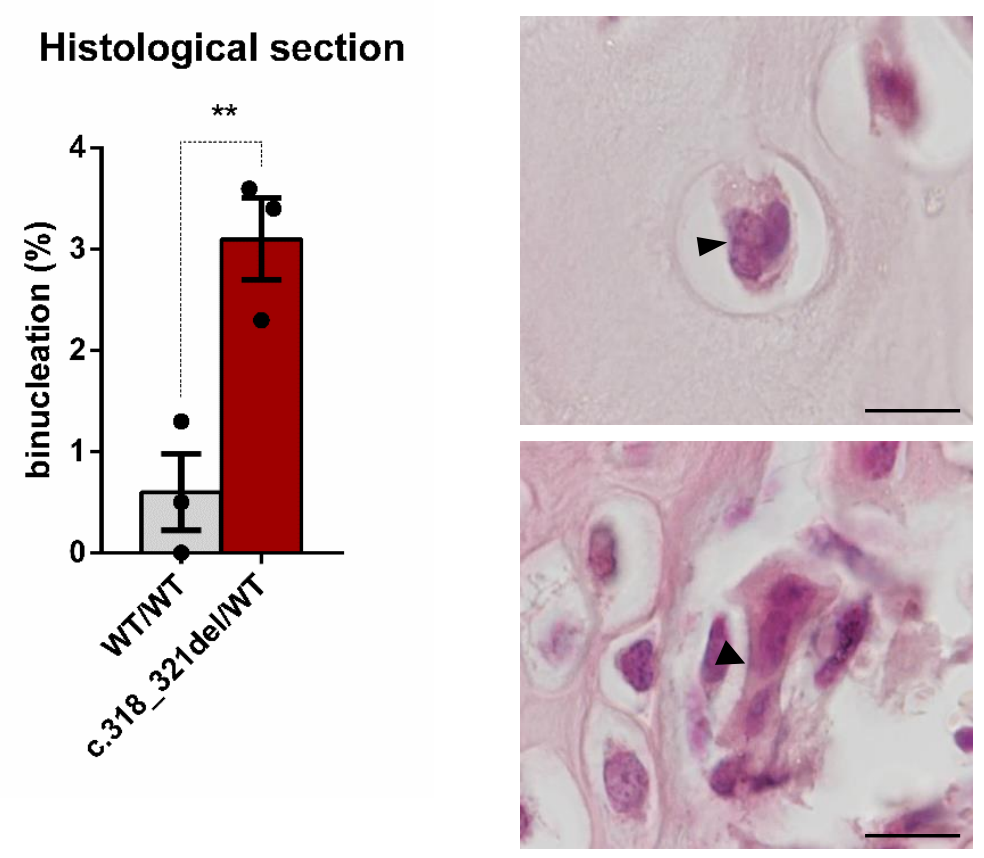
Figure 6: Profilin 1 insufficiency underlies cell division defects in vivo. (a) Representative images of micronuclei (white arrows) in mesenchymal cells isolated from the calvarias of Pfn1 knock-in (c.318_321del/WT) mice; scale bar $10 \mu \mathrm{m}$. (b) Quantification (\%) of micronuclei in normal (WT/WT) and Profilin 1-deficient (c.318_321del/WT) mesenchymal cells; data are shown as mean \pm s.e.m.; dots represent the value of each experiment ( $n=4$ biological replicates for WT samples, 5 biological replicates for mutant samples; scoring a total of 485 WT and 464 mutant cells); ${ }^{*} P=0.0312$. One-tailed unpaired Student's t-tests performed. (c) Quantification (\%) of binucleation in normal (WT/WT) and Profilin 1-deficient (c.318_321del/WT) mesenchymal cells; data are shown as mean \pm s.e.m.; dots represent the value of each experiment $(n=4$ biological replicates for WT samples, 5 biological replicates for mutant samples; scoring a total of 646 WT and 711 mutant cells); ns $P=0.0778$. One-tailed unpaired Student's t-tests performed. (d) Images of primary $P f n 1^{c .318 \_321 d e l / W T}$ mesenchymal cells stained for F-actin (phalloidin, red) and nuclei (Hoechst 33342, blue); scale bars $50 \mu \mathrm{m}$. Arrowheads point towards trinucleated cells. Note that Profilin 1 insufficiency results in large cells with a loose actin cytoskeleton. (e) Representative haematoxylin/eosin-stained sections of the femoral epiphyseal growth plate of 4-month-old control $\left(P f n 1^{W T / W T}\right)$ and knock-in $\left(P f n 1^{c .318 \_321 d e l / W T}\right)$ mice; scale bars $100 \mu \mathrm{m}$. Arrows point towards binucleated cells. (f) Quantification (\%) of binucleated cells in the femoral growth plate of control $\left(P f n 1^{W T / W T}\right)$ and knock-in $\left(P f n 1^{c .318 \_321 d e l / W T}\right)$ mice; data are shown as mean \pm s.e.m.; dots represent the value of each experiment ( $n=3$ biological replicates; scoring $\geq 61$ cells per replicate; $* * P=$ 0.0054. One-tailed unpaired Student's t-tests performed. (g) Haematoxylin/eosin-stained sections showing trinucleated cells (arrowheads) in 4-month-old Pfn1 $1^{c .318 \_321 d e l / W T}$ mice; scale bar $100 \mu \mathrm{m}$. 
bioRxiv preprint doi: https://doi org/10.1101/2022 02 01.478160: this version posted February 1, 2022. The copyright holder for this preprint (which was not certified by peer review) is the author/funder. All rights reserved. No reuse allowed without permission.

Figure 7

a

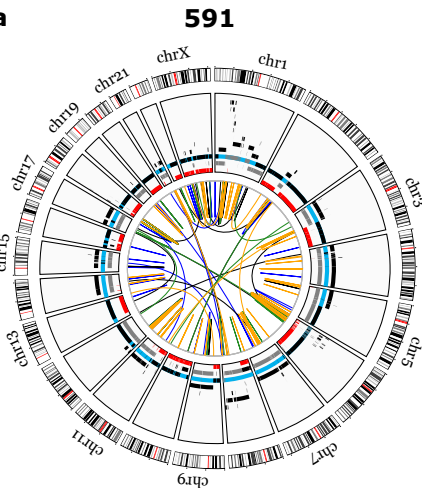

1363

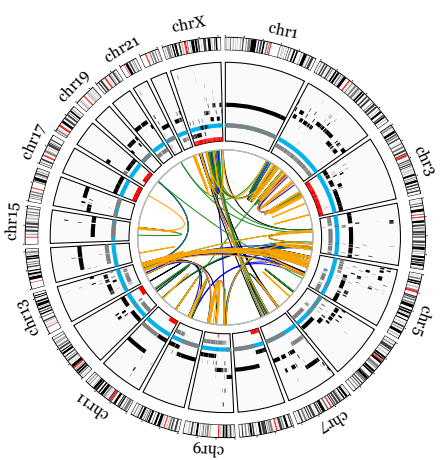

1430

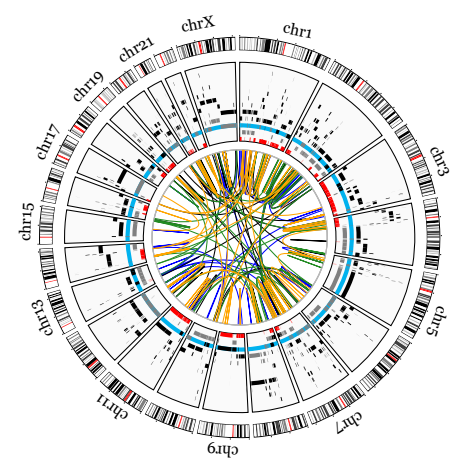

1798

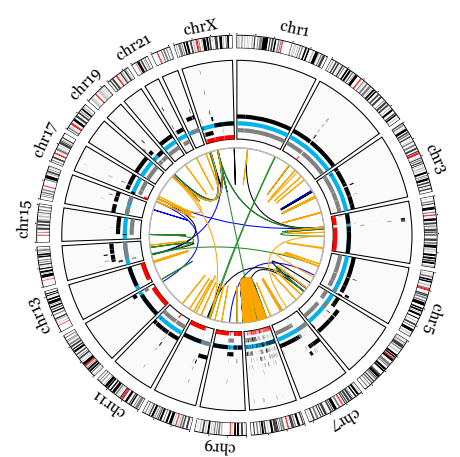

b

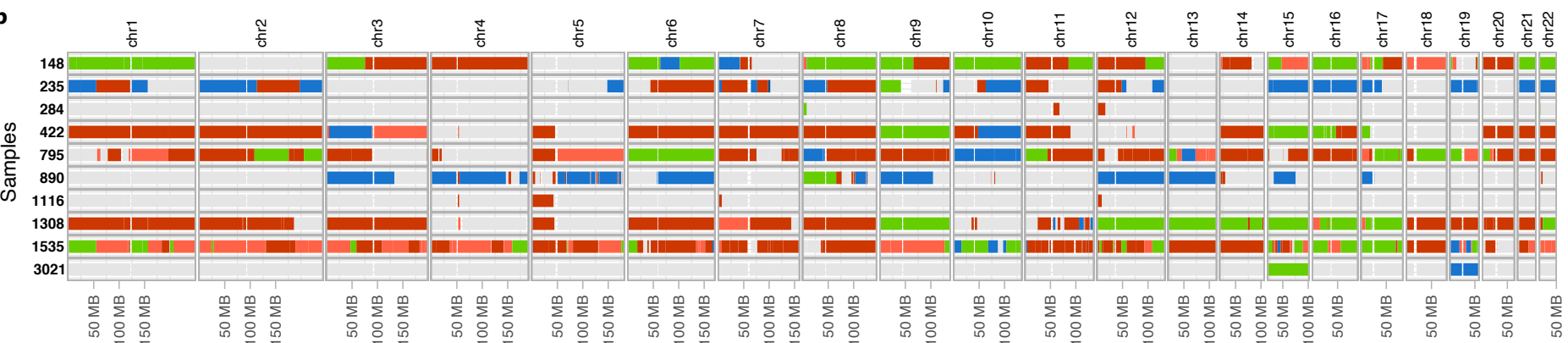

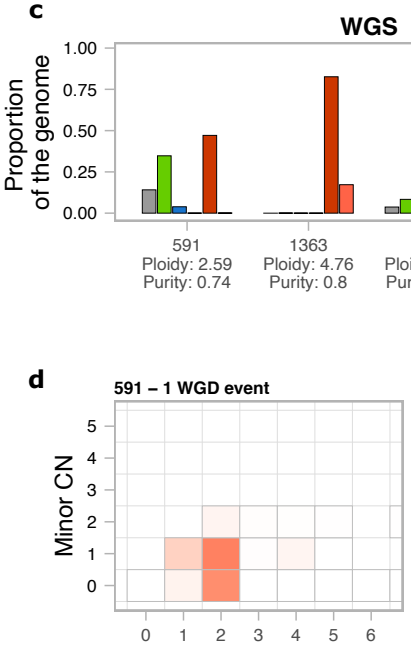

Major CN

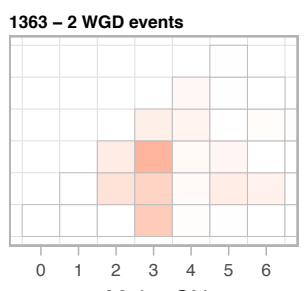

Major CN

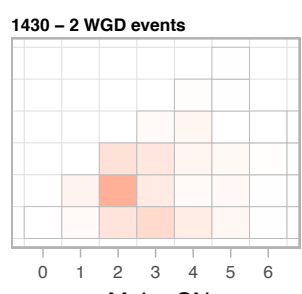

Major CN

WES

Proportion of

the genome $0.2 \quad 0.4>0$

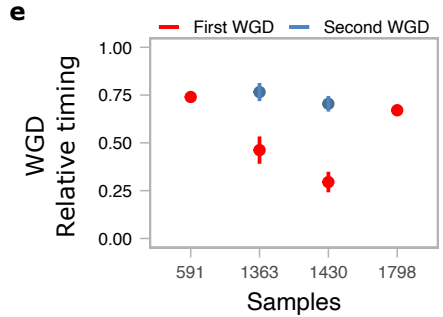


Figure 7. Somatic genomic landscape of 14 OS/PDB patients. (a) Circos plots for 4 OS/PDB patients analysed using whole-genome sequencing. The inner circle shows somatic SVs coloured according to the type of SV (duplication-like, deletion-like, head-to-head and tail-to-tail inversions are shown in blue, orange, black and green, respectively). The second circle shows SCNAs, with black representing the total copy number, grey the minor copy number, and red the minor copy number when equal to 0 . Blue segments mark the expected normal diploid state $(\mathrm{CN}=2)$ as a reference for visualisation. (b) SCNA calls for the 10 WES OS/PDB patients. The colour of the bars represents the different types of SCNAs. (c) Proportion of the genome affected by each type of SCNA. (d) Heatmap of the proportion of the genome representing the different combinations of the obtained major and minor copy numbers in the WGS samples used to estimate the number of WGD events. (e) Relative timing analysis of the WGD events for the WGS samples. The colour of the dots represents the first (red) and second (blue) WGS events for each sample. Lines represent the $95 \%$ confidence intervals after bootstrapping 1,000 times. Neutral: total copy number $=2$ and minor copy number $=1$; Neutral-LoH: total copy number $=2$ and minor copy number $=0$; Hemizygous: total copy number $=1$; Hom-del: homozygous deletion; Amp-LoH: total copy number $>2$ and minor copy number $=0$. 


\section{Description of Additional Supplementary Files}

Supplementary Video 1. Time-lapse of WT RPE1 cells: Phase-contrast live cell imaging of WT RPE1 cells after thymidine synchronisation and release. The majority of mitoses occurred after 4-6 hours of recording (6-8 hours from release in thymidine-free medium). Images were acquired at 2 min intervals over a period of 17 hours and they are played back at 10 frames per second. Timestamp shows relative time in hours:minutes:seconds:milliseconds. Imaging was performed with a 20x objective.

Supplementary Video 2. Time-lapse of $P F N 1^{+/}$RPE1 cells: Phase-contrast live cell imaging of heterozygous PFN1-KO RPE1 cells after thymidine synchronisation and release. The video shows that after 8 hours (10 hours after the release) KO cells attempt to undergo cell division but struggle or fail to round up. Images were acquired at 2 min intervals over a period of 17 hours and they are played back at 10 frames per second. Timestamp shows relative time in hours:minutes:seconds:milliseconds. Imaging was performed with a 20x objective.

Supplementary Video 3. Time-lapse of $P F N 1^{-/-}$RPE1 cells: Phase-contrast live cell imaging of homozygous PFN1-KO RPE1 cells after thymidine synchronisation and release. The video shows that only few cells manage to undergo mitosis; after 10 hours (12 hours after the release) most KO cells struggle or fail to round up. Images were acquired at $2 \mathrm{~min}$ intervals over a period of 17 hours and they are played back at 10 frames per second. Timestamp shows relative time in hours:minutes:seconds:milliseconds. Imaging was performed with a 20x objective.

\section{Supplementary Video 4. Time-lapse of WT H2B-mCherry/EGFP-Tubulin RPE1 cells:}

Fluorescence live cell imaging of WT RPE1 cells expressing H2B-mCherry and EGFP-Tubulin. Left panel: Merge overlay of DNA (H2B-mCherry, red) and microtubules (EGFP-Tubulin, green). Right panel: DNA (H2B-mCherry, red). Images were acquired at 2 min intervals over a period of 19 hours and they are played back at 10 frames per second. Timestamp shows relative time in hours:minutes:seconds. Images taken with 20x objective.

\section{Supplementary Video 5. Time-lapse of $P F N 1^{+/-}$H2B-mCherry/EGFP-Tubulin RPE1 cells:} Fluorescence live cell imaging of heterozygous PFN1-KO RPE1 cells expressing H2B-mCherry and EGFP-Tubulin. Left panel: Merge overlay of DNA (H2B-mCherry, red) and microtubules (EGFP-Tubulin, green). Right panel: DNA (H2B-mCherry, red). The video shows 3 abnormal mitoses: 1) chromosome misalignment on the metaphase plate at 7h:06';2) chromosome misalignment on the metaphase plate at 9h:16' and delayed anaphase onset; 3) delayed anaphase 
onset, formation of an anaphase bridge at 13h:42', and formation of daughter cells containing one micronucleus (15h:06'). Images were acquired at $2 \mathrm{~min}$ intervals over a period of 19 hours and they are played back at 10 frames per second. Timestamp shows relative time in hours:minutes:seconds. Images taken with 20x objective.

\section{Supplementary Videos 6,7 Time-lapse of $P F N 1^{-/-}$H2B-mCherry/EGFP-Tubulin RPE1 cells:}

Fluorescence live cell imaging of homozygous PFN1-KO RPE1 cells expressing H2B-mCherry and EGFP-Tubulin. Left panel: Merge overlay of DNA (H2B-mCherry, red) and microtubules (EGFPTubulin, green). Right panel: DNA (H2B-mCherry, red). The video 6 shows 3 abnormal mitoses: 1) formation of an anaphase bridge at 00h:10' and generation of a micronucleated daughter cell; 2) abnormal cell rounding at $01 \mathrm{~h}: 24$ ', mitotic failure with the extrusion of chromosomes (or chromosome fragments) and formation of nuclear protrusions; 3 ) chromosome misalignment on the metaphase plate at 12h:00' and delayed anaphase onset. Note the dead cells floating in the culture medium. The video 7 shows the formation of a chromosome bridge at $05 \mathrm{~h}: 24$ ' undergoing breakage at 10h:52' and resulting in micronucleated daughter cells. Images were acquired at 2 min intervals over a period of 19 hours and they are played back at 10 frames per second. Timestamp shows relative time in hours:minutes:seconds. Images taken with 20x objective.

Supplementary Video 8. Time-lapse of WT MC3T3 cells: Phase-contrast live cell imaging of WT MC3T3 cells after thymidine synchronisation and release. The majority of mitoses occurred after 4-6 hours of recording (6-8 hours from release in thymidine-free medium); almost all cells divided. Images were acquired at 2 min intervals over a period of 17 hours and they are played back at 10 frames per second. Timestamp shows relative time in hours:minutes:seconds:milliseconds. Imaging was performed with a 20x objective.

Supplementary Video 9. Time-lapse of $\boldsymbol{P f n} \boldsymbol{1}^{+/-}$MC3T3 cells: Phase-contrast live cell imaging of heterozygous $P f n 1^{+-}$MC3T3 cells after thymidine synchronisation and release. The majority of mitoses occurred after 7-9 hours of recording (9-11 hours from release in thymidine-free medium); the video shows that cells make one or more attempts before completely rounding up. Images were acquired at 2 min intervals over a period of 17 hours and they are played back at 10 frames per second. Timestamp shows relative time in hours:minutes:seconds:milliseconds. Imaging was performed with a $20 \mathrm{x}$ objective.

Supplementary Video 10. Time-lapse of WT MEFs: Phase-contrast live cell imaging of WT mouse embryonic fibroblasts. Images were acquired at $2 \mathrm{~min}$ intervals over a period of 16 hours and 
they are played back at 10 frames per second. Timestamp shows relative time in hours:minutes:seconds:milliseconds. Imaging was performed with a 20x objective.

Supplementary Video 11. Time-lapse of Pfn1 $1^{c .318 \_321 d e l / W T}$ MEFs: Phase-contrast live cell imaging of $P f n 1$ heterozygous knock-in mouse embryonic fibroblasts. Yellow arrowheads point towards cells undergoing cytokinesis failure and resulting in single daughter cells with $\geq 2$ nuclei. White arrowheads point towards cells struggling or failing to round up. The asterisk indicates a cell giving rise to three daughter cells after division. Images were acquired at 2 min intervals over a period of 16 hours and they are played back at 10 frames per second. Timestamp shows relative time in hours:minutes:seconds:milliseconds. Imaging was performed with a 20x objective. 
Supplementary Table 1: Analysis of mitotic abnormalities in Profilin 1 knock-out RPE1 cells.

\begin{tabular}{c|cccccc}
$\begin{array}{c}\text { RPE1 } \\
\text { sample }\end{array}$ & $\begin{array}{c}\text { Mitoses } \\
(\boldsymbol{n})\end{array}$ & $\begin{array}{c}\text { Mitotic } \\
\text { defects } \\
(\boldsymbol{n} ; \boldsymbol{\%})\end{array}$ & $\begin{array}{c}\text { Misaligned } \\
\text { chromosomes }\end{array}$ & $\begin{array}{c}\text { Multipolar } \\
\text { spindles } \\
(\boldsymbol{n})\end{array}$ & $\begin{array}{c}\text { Anaphase } \\
\text { bridges } \\
(\boldsymbol{n})\end{array}$ & $\begin{array}{c}\text { Lagging } \\
\text { chromosomes }\end{array}$ \\
\hline Wild type & 911 & $39 ; 4,3 \%$ & 6 & 1 & 31 & 1 \\
PFN1 $^{+-}$ & 825 & $234 ; 28,4 \%$ & 29 & 38 & 148 & 19 \\
PFN1 $^{-/-}$ & 616 & $191 ; 31,0 \%$ & 23 & 26 & 120 & 22
\end{tabular}




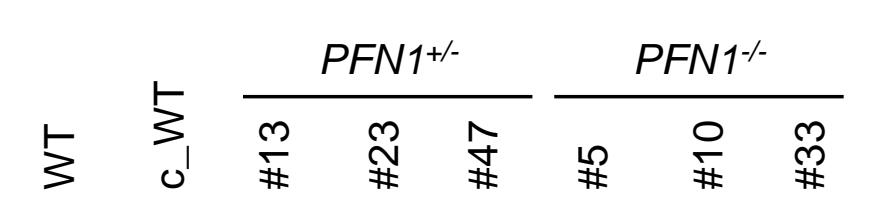

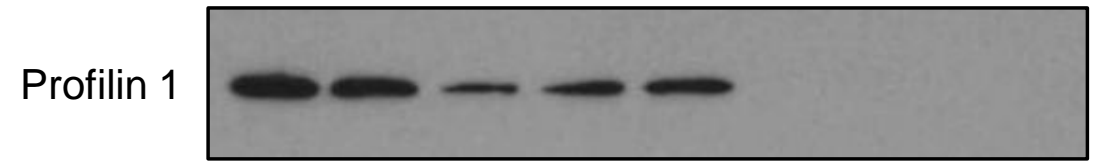

a-Tubulin

b

clone \#13

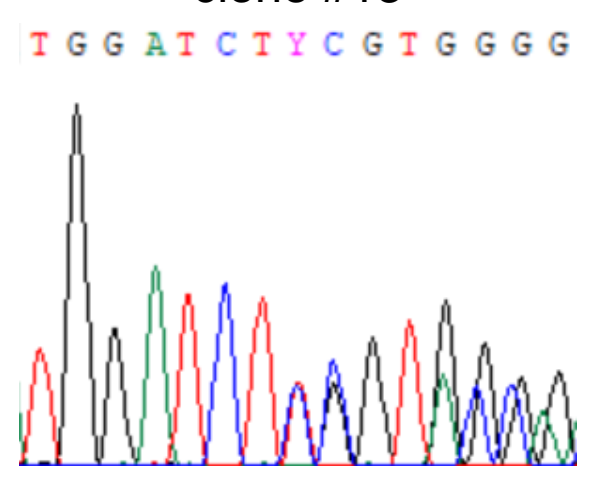

clone \#5

C G I A C A M G R K R S G

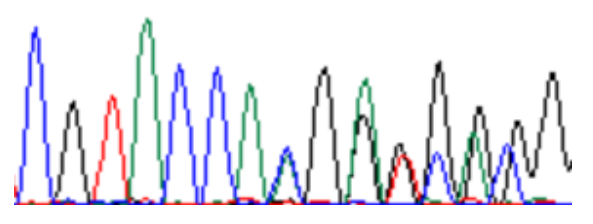

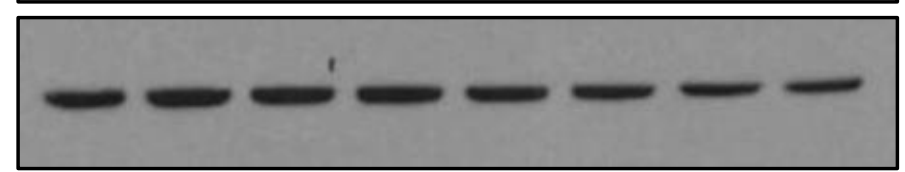

clone \#23

A G C A CS G K K G G G C C

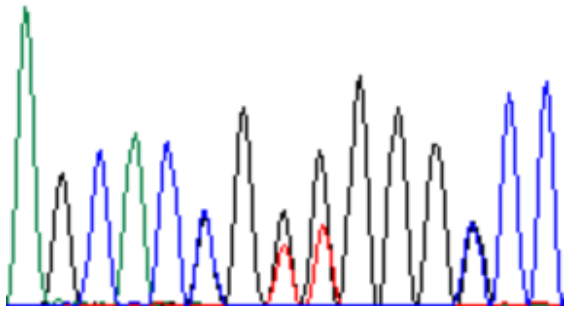

clone \#10

A A G A C A C G G T G G G

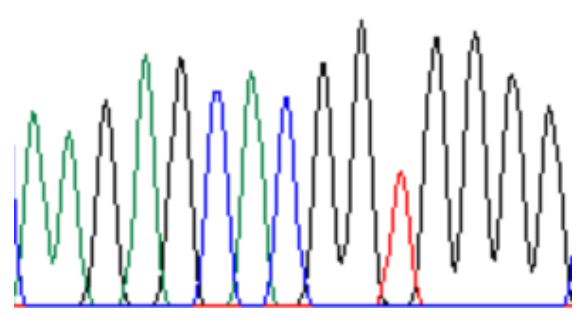

AT G G G G A M K T G G S G

clone \#47

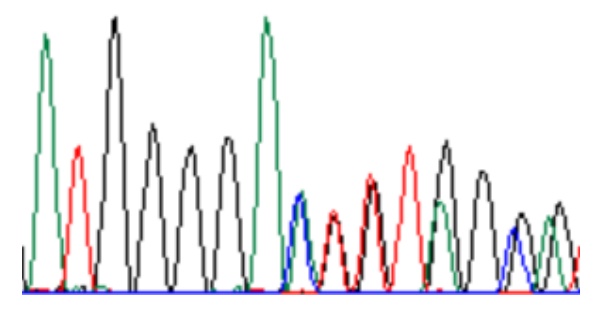

clone \#33

A G A G C A S S K G K G S

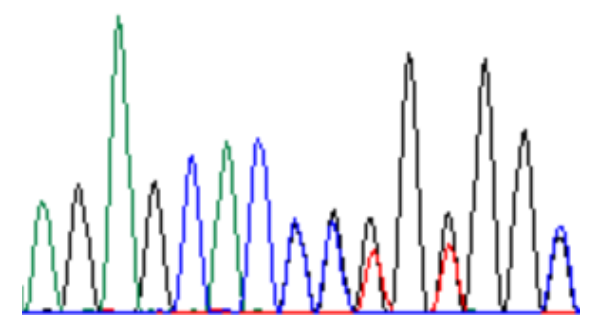

Supplementary Fig. 1: (a) Western blot analysis of Profilin 1 expression in 3 PFN1+/- and 3 PFN1\% RPE1 clones compared with wild type parental cells (WT) and cells subjected to Cas9/gRNA transfection but resulted wild type for PFN1 (c_WT); (b) Sanger sequencing results showing the CRISPR-Cas9-induced INDELs in the indicated RPE1 clones. 


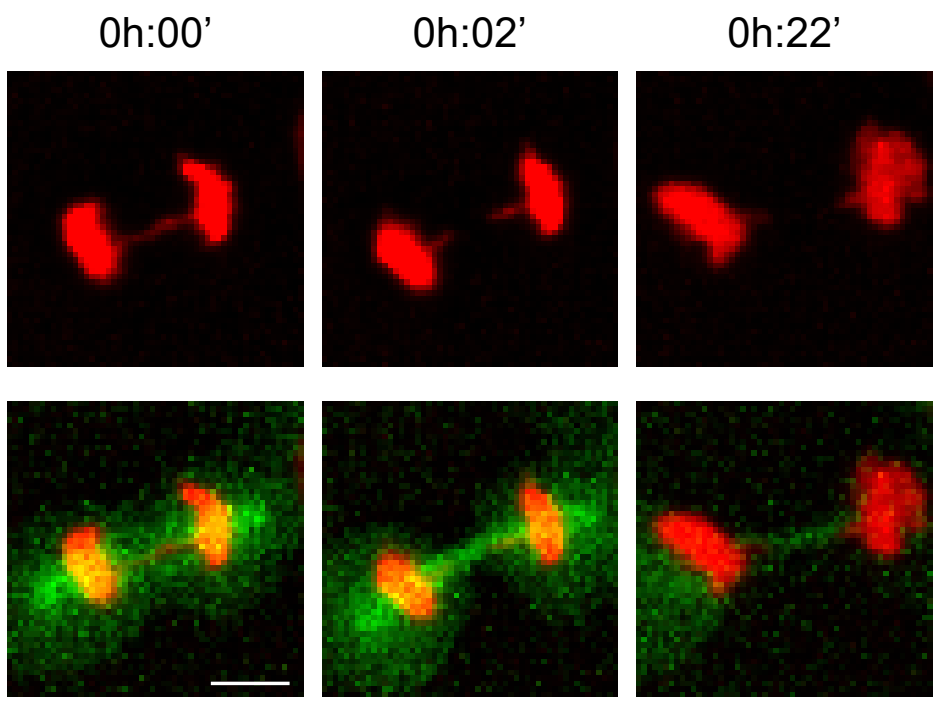

Supplementary Fig. 3: Fluorescence time-lapse microscopy of a PFN1-KO RPE1 cell undergoing mitosis, producing a chromosome bridge that breaks in telophase. Scale bar $10 \mu \mathrm{m}$. DNA is shown in red (H2B-mCherry) and microtubules in green (EGFP-Tubulin). Time stamps indicate elapsed time in hours:minutes. 
a
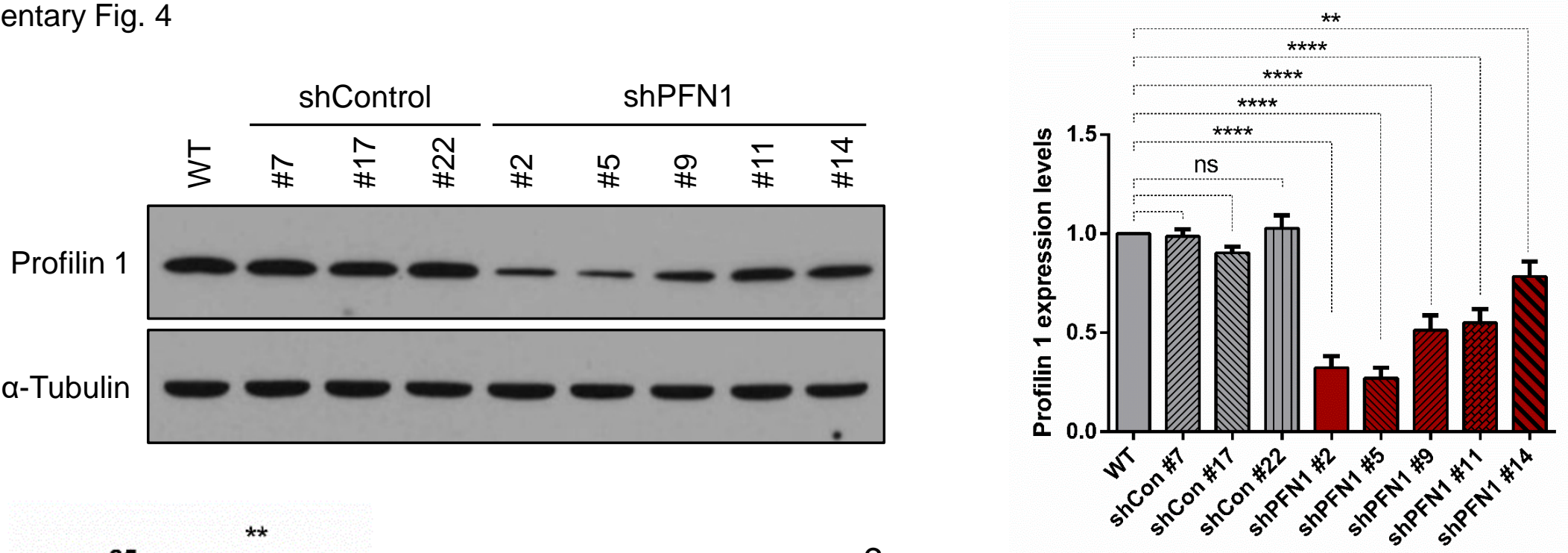

b

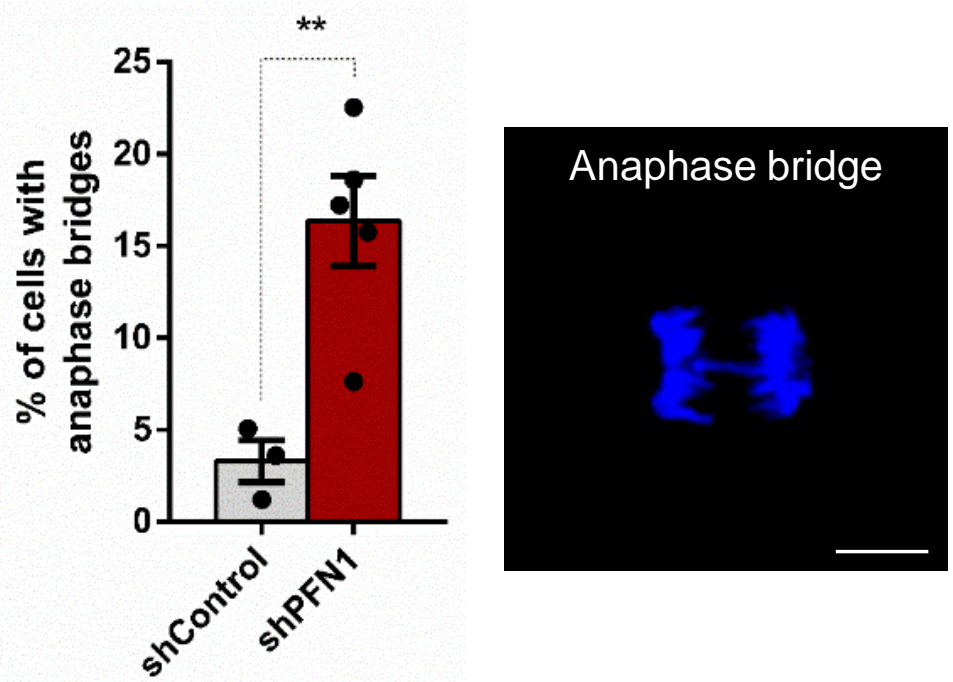

C

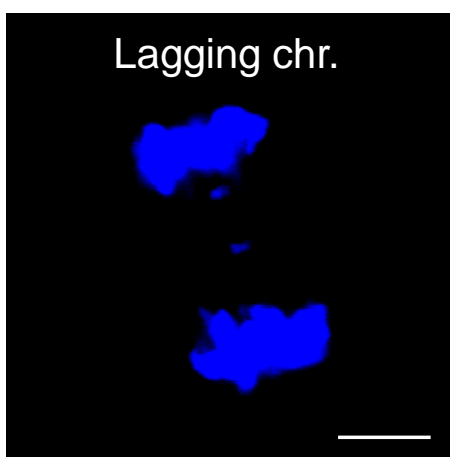

Supplementary Fig. 4: (a) Western blot analysis of Profilin 1 expression in 3 RPE1 clones expressing a non-target shRNA (shControl) and 5 RPE1 clones expressing the shPFN1. On the right, bar graph of band intensity quantification is shown ( $n=3$ independent experiments; $\left.{ }^{\star * \star *} P<0,0001,{ }^{* *} P=0,0013\right)$. Ordinary one-way ANOVA performed. (b) The bar graph shows the quantification of anaphase bridges in RPE1 cells with and without PFN1 knockdown; bars indicate mean \pm s.e.m., dots represent the mean percentage of two independent experiments $(n=3$ biological replicates for shControl, 5 for shPFN1 cells; scoring a total of 183 control and 307 knockdown mitoses); ${ }^{\star \star} P=0,0041$. One-tailed unpaired Student's t-tests performed. On the right, confocal image of Hoechst-stained PFN1 knockdown RPE1 cells showing an anaphase bridge. (c) Representative images of Hoechst-stained PFN1 knockdown RPE1 cells with the indicated mitotic defects. Scale bars $5 \mu \mathrm{m}$. 

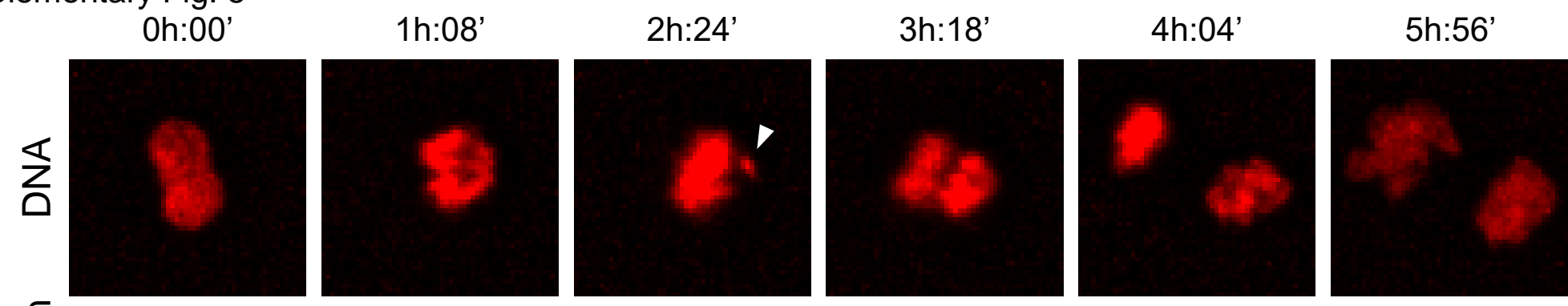

13h:58'
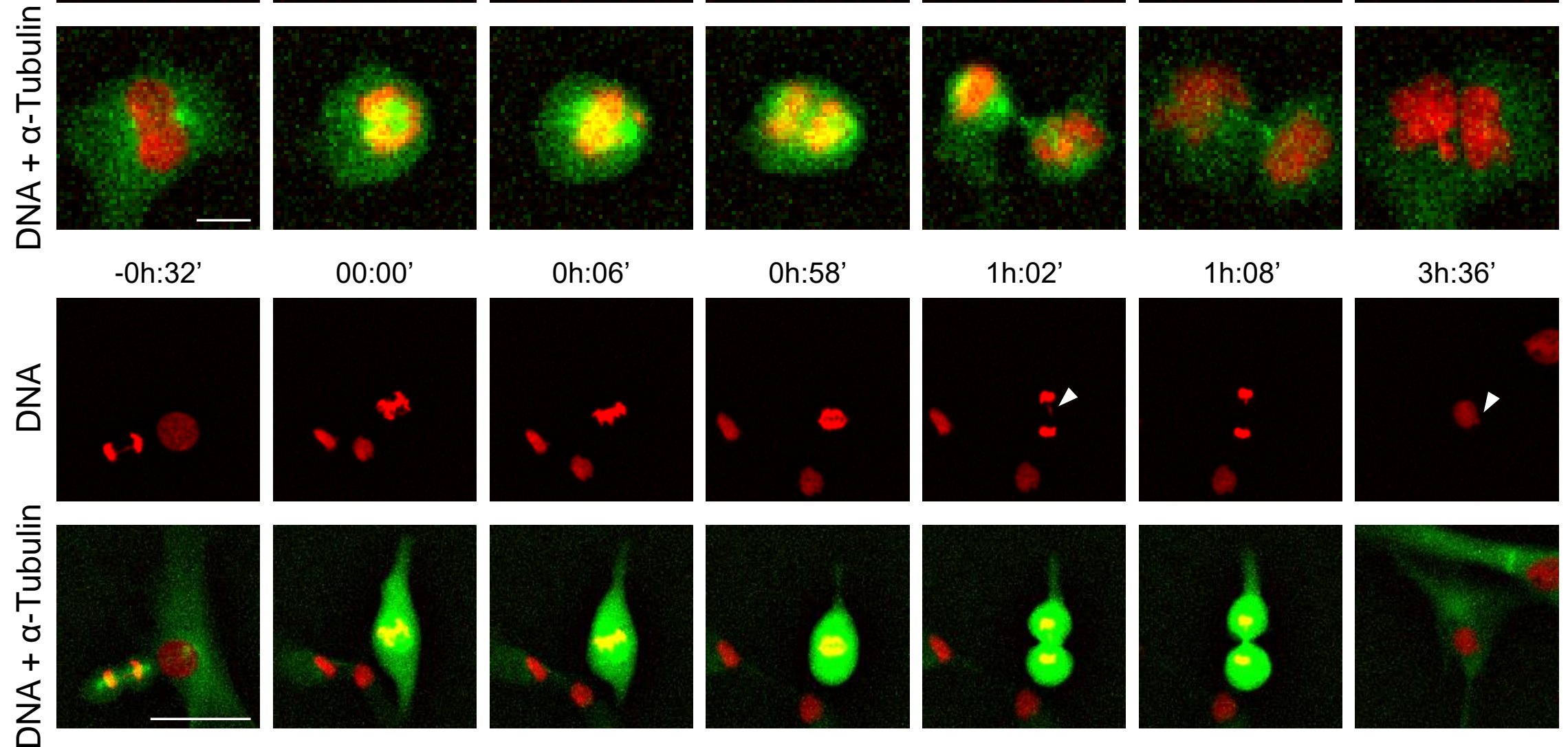

Supplementary Fig. 5: Fluorescence time-lapse microscopy of PFN1-KO RPE1 cells undergoing mitosis. (a) Mitotic KO cell showing a misaligned chromosome in metaphase (arrowhed), delayed anaphase onset, and cytokinesis failure resulting in one daughter cell containing two abnormal nuclei and one micronucleus (arrowhead). Scale bar $10 \mu \mathrm{m}$. (b) Mitotic KO cell starting metaphase without completely rounding up, delaying anaphase onset, and showing a lagging chromosome fragment (arrowhead) that results in an interphase micronucleus (arrowhead) in the daughter cell. Note the chromosome bridge formed in the mitotic cell on the left (first panel). Scale bar $50 \mu \mathrm{m}$. DNA is shown in red (H2B-mCherry) and microtubules in green (EGFP-Tubulin). Time stamps indicate elapsed time in hours:minutes. 
a
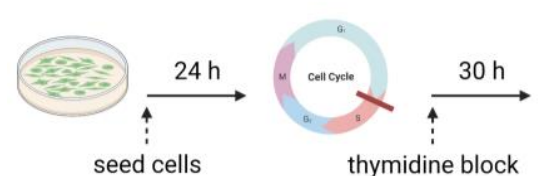

thymidine block
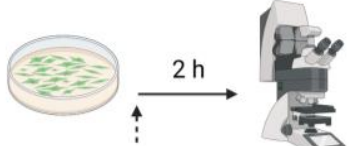

phase contrast live cell imaging

wash and release b

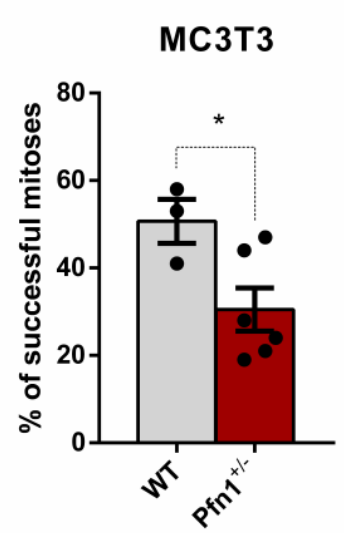

C

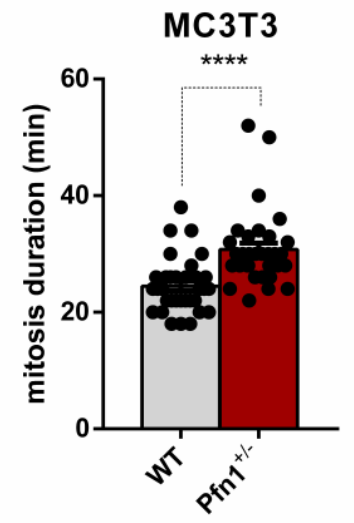

d

$-0 h: 06$

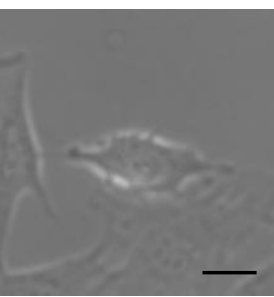

$-0 h: 16$

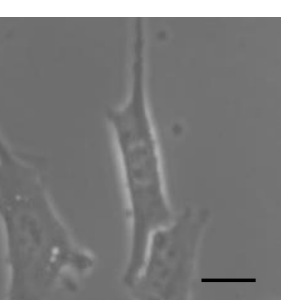

Oh:00'

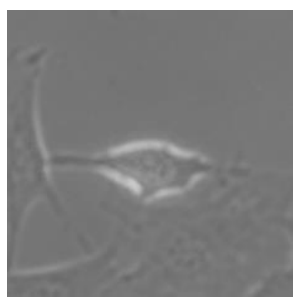

Oh:00'

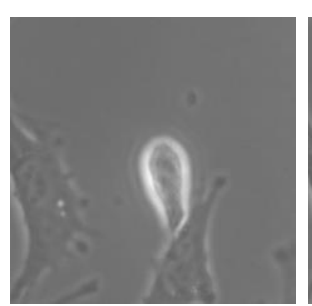

Oh:06'

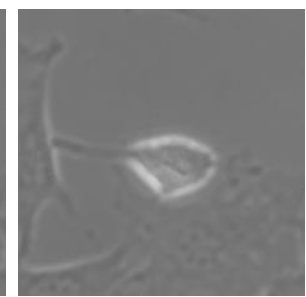

Oh:10'

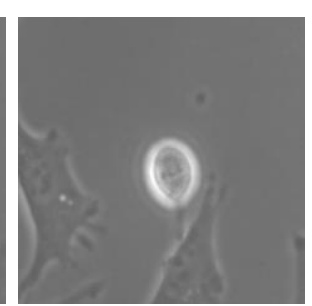

Oh:10'

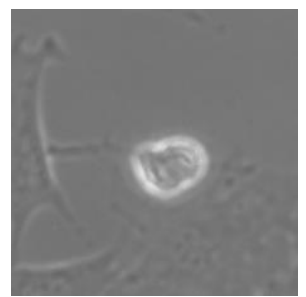

Oh:14'

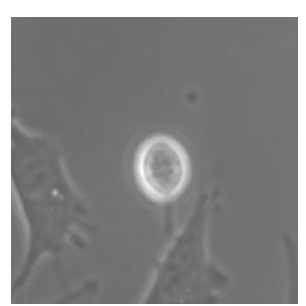

Oh:12'

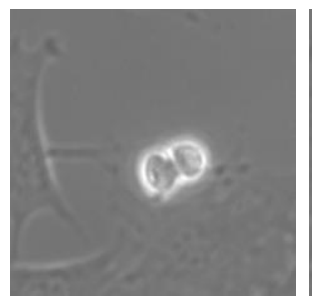

Oh:16'

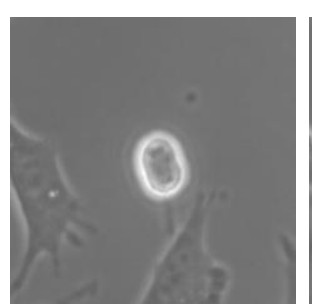

Oh:14'

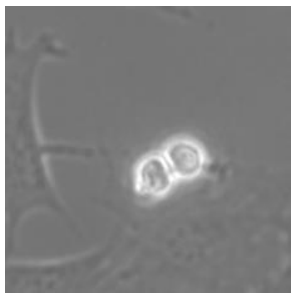

Oh:20'

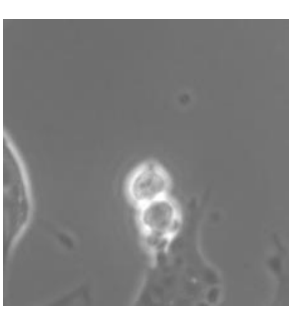

Oh:20'

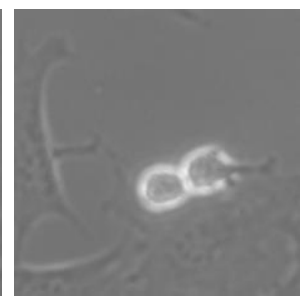

Oh:34'

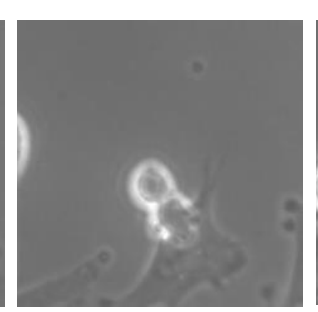

Oh:28'

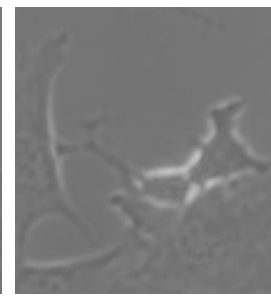

Oh:44'

Supplementary Fig. 6: (a) Schematic of the experiment. (b) Percentage of cells able to complete mitosis following the round up; data are shown as mean \pm s.e.m.; dots represent the mean for each experiment $\left(n=73 \mathrm{WT}, 105\right.$ Pfn $1^{+/-}$MC3T3 cells); ${ }^{*} P=0,0192$. (c) Time taken from start (cell rounding) to end (cytokinesis) of mitosis; data are shown as mean \pm s.e.m.; dots represent the duration of each cell division expressed in minutes ( $n$ mitoses $=33$ WT, 32 Pfn $1^{+/}$MC3T3 cells); ${ }^{* \star * *} P<0,0001$. Data in $\mathbf{b}$ and $\mathbf{c}$ were analysed by one-tailed unpaired Student's $t$-tests. (d) Representative time-lapse phase contrast images (taken every 2 minutes) of WT (top row) and Pfn $1^{+/-}$(bottom row) MC3T3 cells; scale bars $25 \mu \mathrm{m}$. Time stamps indicate elapsed time in hours:minutes. 
Supplementary Fig. 7

a

Pfn1 genomic locus
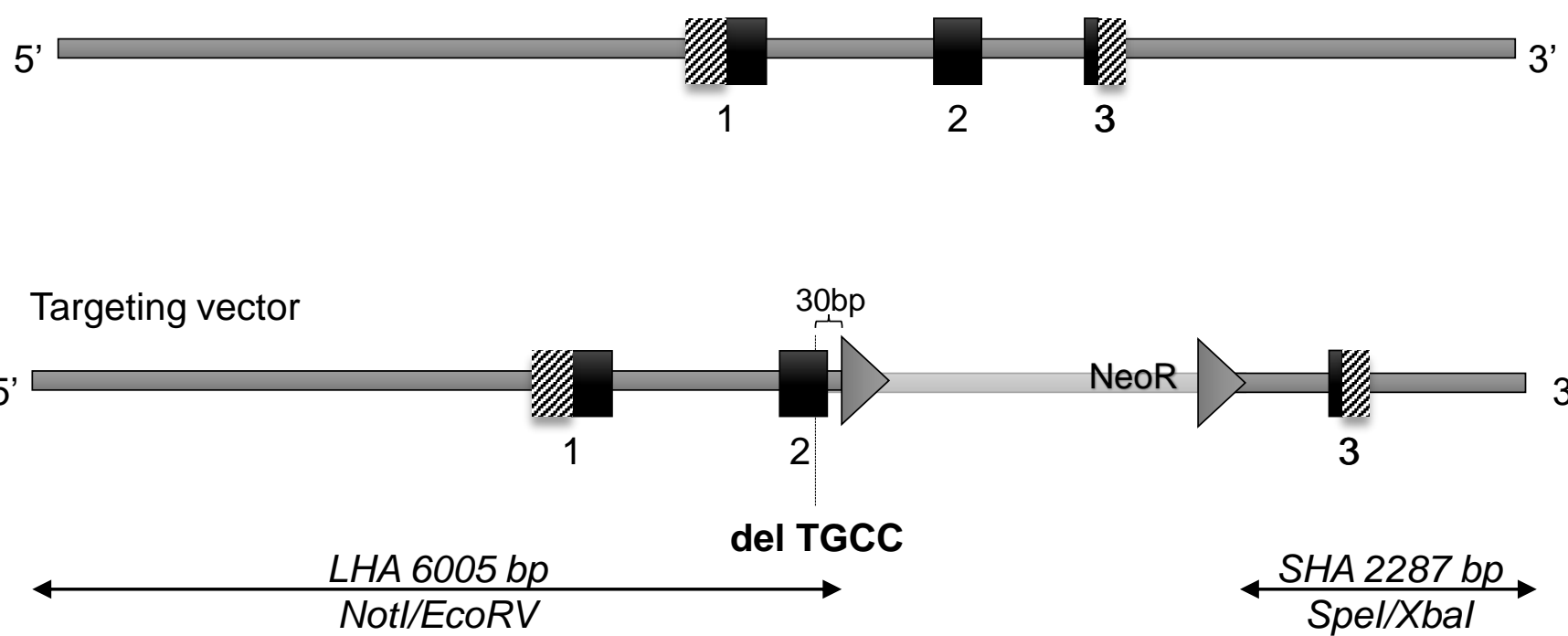

NeoR

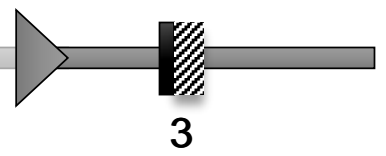

3'

3

LHA 6005 bp

$\frac{S H A 2287 b p}{S p e l / X b a l}$

Mouse genomic region

Coding exon

Untranslated region

LoxP site

LHA: Long Homology Arm SHA: Short Homology Arm

b

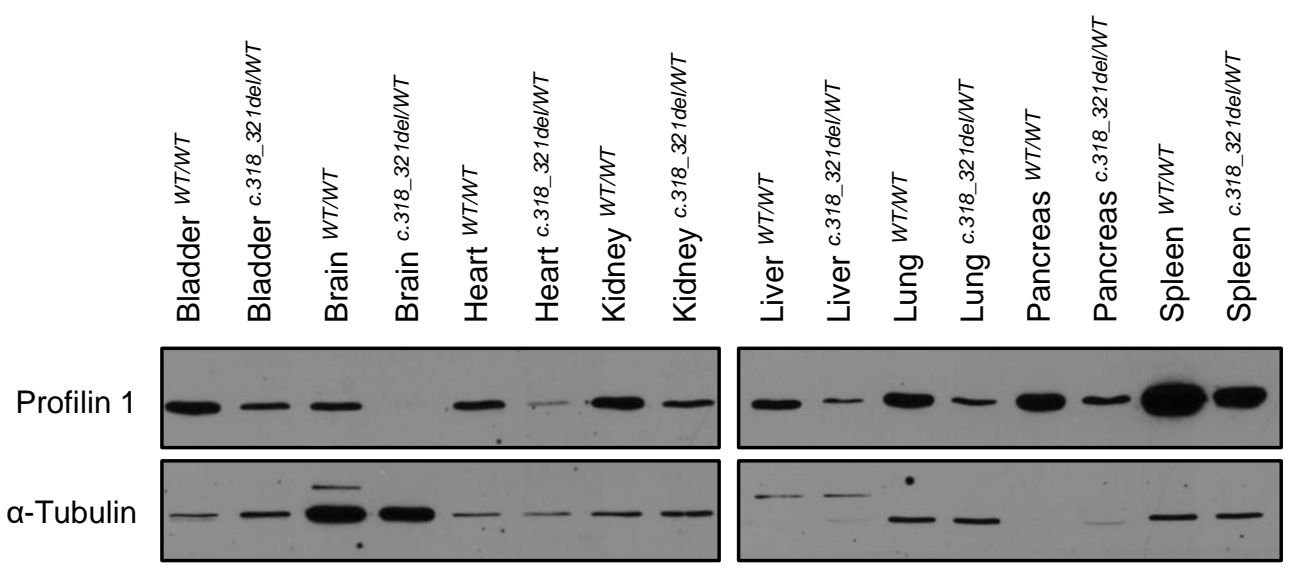

Supplementary Fig. 7: Generation of Pfn1 c.318_321delTGCC constitutive knock-in mouse model. (a) Schematic representation of the wild type Pfn1 locus (upper panel) and targeting strategy (lower panel). The 4-bp deletion was inserted in exon 2; LoxP-flanked Neo cassette was inserted in intron 2. (b) Western blotting analysis of Profilin 1 expression in the indicated mouse tissues; western blot of $\alpha$-Tubulin represents internal controls. WT/WT indicates wild type tissues; c.318_321del/WT indicates tissues from Pfn1 heterozygous knock-in animals. 


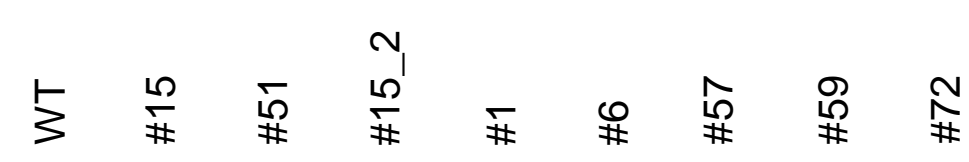

Profilin 1

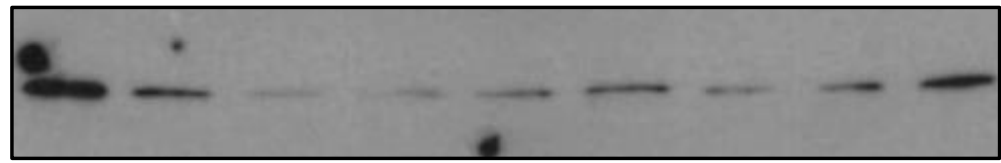

a-Tubulin

b

clone \#15_2

A C C A T G A B K S M S W DD

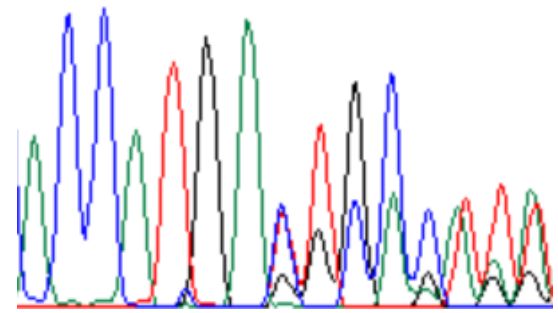

clone \#57

G I CACI G I CA C C A I G

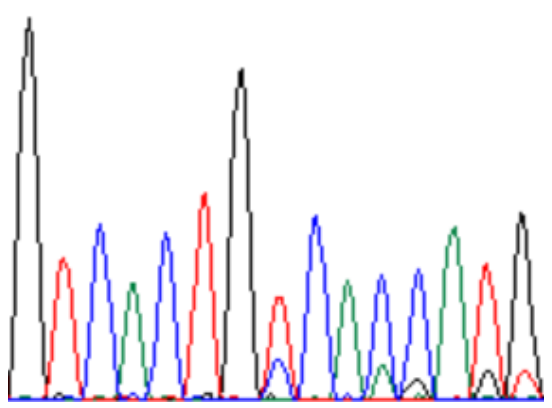

clone \#1

G I C A C C A I G A C I G C C

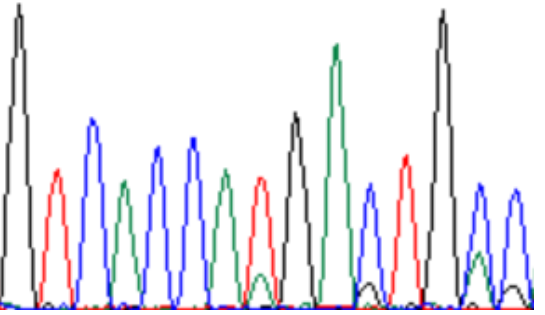

clone \#59

I I CAA I G I CAC I G I C

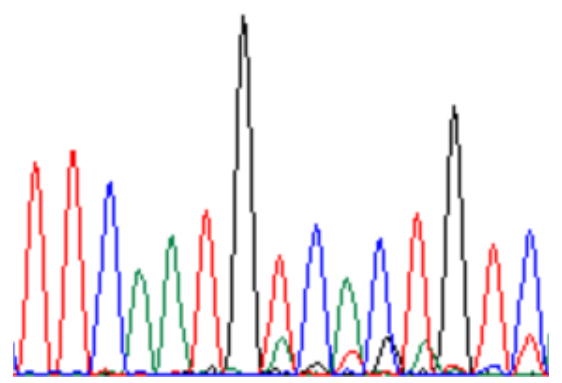

clone \#6

I CACCA I G A C I G C CA

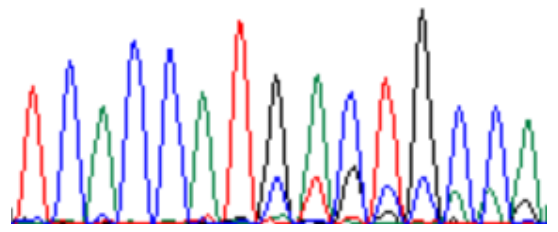

clone \#72

A C CA I G A C I G S CMAR

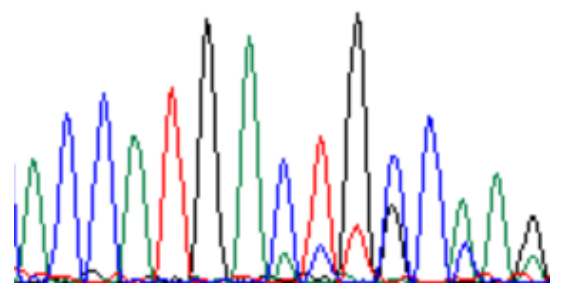

Supplementary Fig. 8: (a) Western blot analysis of Profilin 1 expression in 8 independent heterozygous Pfn1-KO MC3T3 extracts compared with WT cells; (b) Sanger sequencing results showing the CRISPR-Cas9-induced INDELs in the indicated MC3T3 clones. For the electropherogram plots of clones \#15 and \#51, see Ref. 26. 
